\title{
Homotopy transfer and rational models for mapping spaces
}

\author{
Urtzi Buijs $^{1}$ • Javier J. Gutiérrez ${ }^{2}$
}

Received: 1 August 2014 / Accepted: 11 March 2015 / Published online: 28 April 2015

(C) Tbilisi Centre for Mathematical Sciences 2015

\begin{abstract}
By using homotopy transfer techniques in the context of rational homotopy theory, we show that if $C$ is a coalgebra model of a space $X$, then the $A_{\infty}$-coalgebra structure in $H_{*}(X ; \mathbb{Q}) \cong H_{*}(C)$ induced by the higher Massey coproducts provides the construction of the Quillen minimal model of $X$. We also describe an explicit $L_{\infty^{-}}$ structure on the complex of linear maps $\operatorname{Hom}\left(H_{*}(X ; \mathbb{Q}), \pi_{*}(\Omega Y) \otimes \mathbb{Q}\right)$, where $X$ is a finite nilpotent $\mathrm{CW}$-complex and $Y$ is a nilpotent $\mathrm{CW}$-complex of finite type, modeling the rational homotopy type of the mapping space $\operatorname{map}(X, Y)$. As an application we give conditions on the source and target in order to detect rational $H$-space structures on the components.
\end{abstract}

Keywords Rational homotopy $\cdot L_{\infty}$-algebra $\cdot$ Mapping space

Mathematics Subject Classification Primary 55P62; Secondary 54C35

Communicated by Pascal Lambrechts.

The authors were supported by the MINECO Grant MTM2010-15831 and by the Generalitat de Catalunya as members of the team 2009 SGR 119. The first-named author was supported by a U-Mobility Programme grant of the European Union's Seventh Framework Programme, G.A. no. 246550 and by the Junta de Andalucía Grant FQM-213. The second-named author was supported by the NWO (SPI 61-638).

$\bowtie \quad$ Urtzi Buijs

ubuijs@uma.es

Javier J. Gutiérrez

j.gutierrez@math.ru.nl

1 Departamento de Álgebra, Geometría y Topología, Universidad de Málaga, Ap. 59, 29080 Málaga, Spain

2 Institute for Mathematics, Astrophysics, and Particle Physics, Radboud Universiteit Nijmegen, Heyendaalseweg 135, 6525 AJ Nijmegen, The Netherlands 


\section{Introduction}

The version up to homotopy of differential graded Lie algebras, called $L_{\infty}$-algebras, had its origins in the context of deformation theory [32] and has been highly used since then in different geometrical settings [12,24]. Recently, the theory of $L_{\infty}$ algebras has become a useful tool in order to describe rational homotopy types of spaces $[10,18]$. In particular, mapping spaces seem to be well described in these terms $[3,5,9,11,27]$.

More concretely, if $C$ is a coalgebra model of a finite nilpotent CW-complex $X$ and $L$ is an $L_{\infty}$-model of a finite type rational $C W$-complex $Y$, then the complex of linear maps $\operatorname{Hom}(C, L)$ admits an $L_{\infty}$-structure whose geometrical realization is of the rational homotopy type of $\operatorname{map}(X, Y)$, the space of continuous functions; see [3,9] for details. Similarly, the complex $\operatorname{Hom}(\bar{C}, L)$, where $\bar{C}$ is the kernel of the augmentation $\varepsilon: C \longrightarrow \mathbb{Q}$ is an $L_{\infty}$-model for $\operatorname{map}^{*}(X, Y)$, the space of pointed continuous functions.

The aim of this paper is to describe an explicit $L_{\infty}$-structure in the complex of linear maps $\operatorname{Hom}\left(H_{*}(X ; \mathbb{Q}), \pi_{*}(\Omega Y) \otimes \mathbb{Q}\right)$ that serves as a model for the rational homotopy type of $\operatorname{map}(X, Y)$.

If $L$ denotes the $L_{\infty}$-algebra such that $\mathcal{C}^{*}(L)=(\Lambda V, d)$ is the Sullivan minimal model of $Y$ (see Sect. 2 for details), then we have that $L \cong \pi_{*}(\Omega Y) \otimes \mathbb{Q}$ as graded vector spaces. Let $C$ be a coalgebra model of a finite nilpotent $C W$-complex $X$. The strategy will be to use the homotopy retract between $C$ and $H_{*}(C) \cong H_{*}(X ; \mathbb{Q}$ ) (denoted by $H$ from now on), defining the higher Massey coproducts, and the above explicit $L_{\infty}$-structure on $\operatorname{Hom}(C, L)$ to induce another homotopy retract between $\operatorname{Hom}(C, L)$ and $\operatorname{Hom}(H, L)$. Then, we apply the homotopy transfer theorem $[17,25,26,28,30]$ to give an $L_{\infty}$-structure on $\operatorname{Hom}(H, L)$ and an explicit formula for it by means of rooted trees (see Sect. 3 for further details).

We prove that, indeed, this $L_{\infty}$-structure exhibits $\operatorname{Hom}(H, L)$ as an $L_{\infty}$-model for $\operatorname{map}(X, Y)$. Note, however, that a quasi-isomorphism between two $L_{\infty}$-algebras is not necessarily a quasi-isomorphism after applying the generalized cochain functor $\mathcal{C}^{*}$. Therefore, we cannot deduce directly from the quasi-isomorphism provided by the homotopy retract that $\operatorname{Hom}(H, L)$ is an $L_{\infty}$-model for the mapping space. The same argument can be applied by replacing $(C, \Delta)$ and $H$ with $(\bar{C}, \bar{\Delta})$ and $\bar{H}$, respectively, to model the space of pointed continuous functions map* $(X, Y)$.

Moreover, the homotopy retract between $C$ and $H_{*}(X ; \mathbb{Q})$ used above has its own interest since we show that it provides the construction of the Quillen minimal model of $X$; see Theorem 3.1.

Finally, using the model for map* $(X, Y)$, we give a necessary condition for the components of mapping spaces to be of the rational homotopy type of an $H$-space. This problem has been previously considered in $[5,7,16]$. We prove a variant of the results obtained in these papers in terms of the cone length (cl), the Whitehead length (Wl) and the bracket length (bl), that does not implicitly assume the coformality of the target space. Explicitly, we prove that if $\operatorname{cl}(X)=2$ and $\mathrm{Wl}(Y)<\mathrm{bl}(X)$, then all the components of map* $(X, Y)$ are rationally $H$-spaces. 


\section{Preliminaries}

We will rely on known results from rational homotopy theory for which [15] is a standard reference. We also assume the reader is aware of the concepts of homotopy operadic algebras being [28] an excellent reference. With the aim of fixing notation we give some definitions and sketch some results we will need. Every algebraic object considered throughout the paper is assumed to be a graded vector space over the rationals.

\section{1 $A_{\infty}$-coalgebras and $L_{\infty}$-algebras}

An $A_{\infty}$-coalgebra $C$ is a graded vector space together with a differential graded algebra structure on the tensor algebra $T^{+}\left(s^{-1} C\right)$ on the desuspension of $C$. This is equivalent to the existence of a family of degree $k-2$ linear maps $\Delta_{k}: C \longrightarrow C^{\otimes k}$ satisfying the equation

$$
\sum_{k=1}^{i} \sum_{n=0}^{i-k}(-1)^{k+n+k n}\left(\mathrm{id}^{\otimes i-k-n} \otimes \Delta_{k} \otimes \mathrm{id}^{n}\right) \Delta_{i-k+1}=0 .
$$

Any differential graded coalgebra $(C, \delta, \Delta)$ is an $A_{\infty}$-coalgebra with $\Delta_{1}=\delta, \Delta_{2}=\Delta$ and $\Delta_{k}=0$ for $k>2$. We will denote by $\Delta^{(k)}=(\Delta \otimes \mathrm{id} \otimes \cdots \otimes \mathrm{id}) \circ \cdots \circ(\Delta \otimes \mathrm{id}) \circ \Delta$ with $k$ factors, and $\Delta^{(0)}=$ id.

An $A_{\infty}$-coalgebra is cocommutative if $\tau \circ \Delta_{k}=0$ for every $k \geq 1$, where $\tau: T(C) \longrightarrow T(C) \otimes T(C)$ denotes the unshuffle coproduct, that is,

$\tau\left(a_{1} \otimes \cdots \otimes a_{n}\right)=\sum_{i=1}^{n} \sum_{\sigma \in S(i, n-i)} \epsilon_{\sigma}\left(a_{\sigma(1)} \otimes \cdots \otimes a_{\sigma(i)}\right) \otimes\left(a_{\sigma(i+1)} \otimes \cdots \otimes a_{\sigma(n)}\right)$,

where $\epsilon_{\sigma}$ is the signature of $\sigma$ and $S(i, n-i)$ denotes the set of $(i, n-i)$-shuffles, i.e., permutations $\sigma$ of $n$-elements such that $\sigma(1)<\cdots<\sigma(i)$ and $\sigma(i+1)<\cdots<\sigma(n)$.

Let $\left(C,\left\{\Delta_{k}\right\}\right)$ and $\left(C^{\prime},\left\{\Delta_{k}^{\prime}\right\}\right)$ be two $A_{\infty}$-coalgebras. A morphism of $A_{\infty}$-coalgebras from $C$ to $C^{\prime}$ is a morphism $f: C \rightarrow C^{\prime}$ compatible with $\Delta_{k}$ and $\Delta_{k}^{\prime}$. An $A_{\infty}$-morphism from $C$ to $C^{\prime}$ is a morphism $f: T^{+}\left(s^{-1} C\right) \rightarrow T^{+}\left(s^{-1} C^{\prime}\right)$ of differential graded algebras. This is equivalent to the existence of a family of degree $k-1$ maps $f^{(k)}: C \rightarrow C^{\prime \otimes k}$ satisfying the usual relations involving $\Delta_{k}$ and $\Delta_{k}^{\prime}$. An $A_{\infty}$-morphism is a quasi-isomorphism if $f^{(1)}$ is a quasi-isomorphism of complexes.

An $L_{\infty}$-algebra or strongly homotopy Lie algebra is a graded vector space $L$ together with a differential graded coalgebra structure on $\Lambda^{+}{ }_{s} L$, the cofree graded cocommutative coalgebra generated by the suspension. The existence of this structure on $\Lambda^{+} s L$ is equivalent to the existence of degree $k-2$ linear maps $\ell_{k}: L^{\otimes k} \rightarrow L$, for $k \geq 1$, satisfying the following two conditions:

(i) For any permutation $\sigma$ of $k$ elements,

$$
\ell_{k}\left(x_{\sigma(1)}, \ldots, x_{\sigma(k)}\right)=\epsilon_{\sigma} \epsilon \ell_{k}\left(x_{1}, \ldots, x_{k}\right),
$$


where $\epsilon_{\sigma}$ is the signature of the permutation and $\epsilon$ is the sign given by the Koszul convention.

(ii) The generalized Jacobi identity holds, that is

$$
\sum_{i+j=n+1} \sum_{\sigma \in S(i, n-i)} \epsilon_{\sigma} \epsilon(-1)^{i(j-1)} \ell_{n-i}\left(\ell_{i}\left(x_{\sigma(1)}, \ldots, x_{\sigma(i)}\right), x_{\sigma(i+1)}, \ldots, x_{\sigma(n)}\right)=0,
$$

where $S(i, n-i)$ denotes the set of $(i, n-i)$-shuffles.

Every differential graded Lie algebra $(L, \partial)$ is an $L_{\infty}$-algebra by setting $\ell_{1}=\partial$, $\ell_{2}=[-,-]$ and $\ell_{k}=0$ for $k>2$. An $L_{\infty}$-algebra $\left(L,\left\{\ell_{k}\right\}\right)$ is called minimal if $\ell_{1}=0$.

Let $\left(L,\left\{\ell_{k}\right\}\right)$ and $\left(L^{\prime},\left\{\ell_{k}^{\prime}\right\}\right)$ be two $L_{\infty}$-algebras. A morphism of $L_{\infty}$-algebras from $L$ to $L^{\prime}$ is a morphism $f: L \rightarrow L^{\prime}$ that is compatible with $\ell_{k}$ and $\ell_{k}^{\prime}$. An $L_{\infty^{-}}$ morphism from $L$ to $L^{\prime}$ is a morphism $f: \Lambda^{+} s L \rightarrow \Lambda^{+}{ }_{s} L^{\prime}$ of differential graded coalgebras.

Any $L_{\infty}$-morphism is completely determined by the projection $\pi f: \Lambda^{+} s L \rightarrow s L^{\prime}$ which is the sum of a system of skew-symmetric morphisms $f^{(k)}: L^{\otimes k} \rightarrow L^{\prime}$ of degree $1-k$. The morphisms $f^{(k)}$ satisfy an infinite sequence of equations involving the brackets $\ell_{k}$ and $\ell_{k}^{\prime}$; see for example [24]. In particular, for $k=1$, we have that $\ell_{1}^{\prime} f^{(1)}-f^{(1)} \ell_{1}=0$. Therefore $f^{(1)}:\left(L, \ell_{1}\right) \rightarrow\left(L^{\prime}, \ell_{1}^{\prime}\right)$ is a map of complexes. Any morphism of $L_{\infty}$-algebras is an $L_{\infty}$-morphism of $L_{\infty}$-algebras. As in the case of $A_{\infty}$ coalgebras, an $L_{\infty}$-morphism is a quasi-isomorphism if $f^{(1)}$ is a quasi-isomorphism of complexes. The following result can be found in [24, Theorem 4.6].

Theorem 2.1 Let $L$ and $L^{\prime}$ be two $L_{\infty}$-algebras. If $f$ is a quasi-isomorphism of $L_{\infty}$ algebras from $L$ to $L^{\prime}$, then there exists another $L_{\infty}$-morphism from $L^{\prime}$ to $L$ inducing the inverse isomorphism between homology of complexes $\left(L, \ell_{1}\right)$ and $\left(L^{\prime}, \ell_{1}^{\prime}\right)$.

An $L_{\infty}$-algebra $\left(L,\left\{\ell_{k}\right\}\right)$ is called linear contractible if $\ell_{k}=0$ for $k \geq 2$ and $H_{*}\left(L, \ell_{1}\right)=0$. Since the property of being linear contractible is not invariant under $L_{\infty}$-isomorphisms, we say that $L$ is contractible if $L$ is isomorphic as an $L_{\infty}$-algebra to a linear contractible one. As stated in [24, Lemma 4.9], any $L_{\infty}$-algebra is $L_{\infty^{-}}$ isomorphic to the direct sum of a minimal $L_{\infty}$-algebra and a linear contractible one. Following [24], one can prove that any quasi-isomorphism between minimal $L_{\infty}$ algebras is an isomorphism. Thus, the set of equivalence classes of $L_{\infty}$-algebras up to quasi-isomorphisms can be identified with the set of equivalence classes of minimal $L_{\infty}$-algebras up to $L_{\infty}$-isomorphisms.

The Maurer-Cartan set of an $L_{\infty}$-algebra $L$ is the set of elements $z \in L_{-1}$ such that the infinite series

$$
\sum_{k \geq 1} \frac{1}{k !} \ell_{k}(z, \stackrel{(k)}{.}, z)
$$

is a finite sum and it is equal to zero. We will denote the set of Maurer-Cartan elements in $L$ by $\operatorname{MC}(L)$. If $L$ is an $L_{\infty}$-algebra and $z \in \mathrm{MC}(L)$, then 


$$
\ell_{k}^{z}\left(x_{1}, \ldots, x_{k}\right)=\sum_{i \geq 0} \frac{1}{i !} \ell_{i+k}\left(z, \stackrel{(i)}{.}, z, x_{1}, \ldots, x_{k}\right)
$$

defines a new $L_{\infty}$-structure denoted by $L^{z}$ whenever the series is a finite sum (cf. [18, Proposition 4.4]). The perturbed and truncated $L_{\infty}$-structure on $L$ is the $L_{\infty}$-algebra $L^{(z)}$, whose underlying graded vector space is given by

$$
\left(L^{(z)}\right)_{i}=\left\{\begin{array}{cl}
L_{i} & \text { if } i> \\
Z_{\ell_{1}^{z}}\left(L_{0}\right) & \text { if } i=0 \\
0 & \text { if } i<0
\end{array}\right.
$$

where $Z_{\ell_{1}^{z}}\left(L_{0}\right)$ denotes the space of cycles for the differential $\ell_{1}^{z}$, and with the same brackets as $L^{z}$.

Remark 2.2 If we want to extend the definition of the classical cochain functor $\mathcal{C}^{*}$ $[15, \S 23]$ from the category of differential graded Lie algebras to the category of $L_{\infty}$-algebras, in order to define a realization functor for $L_{\infty}$-algebras as the composition of the cochain functor and Sullivan's realization functor [15, §17], then we should constrain the category of $L_{\infty}$-algebras. For a discussion of the exact technical requirements needed with definitions and examples we refer to $[8, \S 1]$. Since all the $L_{\infty}$-algebras involved in the next sections are of this type of mild $L_{\infty}$-algebras we consider from now on that all $L_{\infty}$-algebras are of this kind.

Then we have that an $L_{\infty}$-algebra structure on $L$ is the same as a commutative differential graded algebra structure on $\Lambda(s L)^{\sharp}$, denoted by $\mathcal{C}^{*}(L)$, where $\sharp$ stands for the dual vector space. More explicitly, if $V$ and $s L$ are dual graded vector spaces, then $\mathcal{C}^{*}(L)=(\Lambda V, d)$ with $d=\sum_{j \geq 1} d_{j}$ and

$$
\left\langle d_{j} v ; s x_{1} \wedge \cdots \wedge s_{j}\right\rangle=(-1)^{\epsilon}\left\langle v ; s \ell_{j}\left(x_{1}, \ldots, x_{j}\right)\right\rangle,
$$

where $\langle-;-\rangle$ is defined as an extension of the pairing induced by the isomorphism between $V$ and $(s L)^{\sharp}, d_{j} v \subset \Lambda^{j} V$ and $\epsilon$ is the sign given by the Koszul convention.

Conversely, if $(\Lambda V, d)$ is a commutative differential graded algebra of finite type, then an $L_{\infty}$-algebra structure on $s^{-1} V^{\sharp}$ is uniquely determined by the condition $(\Lambda V, d)=\mathcal{C}^{*}(L)$.

\subsection{The homotopy transfer theorem}

In this section we recall how to transfer $A_{\infty}$ - and $L_{\infty}$-structures along homotopy retracts. This will be the main tool used in Sect. 3 to describe an explicit $L_{\infty}$-structure on the complex of linear maps $\operatorname{Hom}\left(H_{*}(X ; \mathbb{Q}), \pi_{*}(\Omega Y) \otimes \mathbb{Q}\right)$.

Let $\left(A, d_{A}\right)$ and $\left(V, d_{V}\right)$ be two complexes. We say that $V$ is a homotopy retract of $A$ if there exist maps

$$
h \bigcirc_{A}\left(A, d_{A}\right) \underset{i}{\stackrel{p}{\rightleftarrows}}\left(V, d_{V}\right)
$$


such that $\mathrm{id}_{A}-i p=d_{A} h+h d_{A}$ and $i$ is a quasi-isomorphism. If $(A, V, i, p, h)$ is a homotopy retract, then it is possible to transfer $A_{\infty}$ - and $L_{\infty}$-structures from $A$ to $V$ with explicit formulae. This is in fact a particular instance of the so-called homotopy transfer theorem $[17,25,26,28,30]$, which goes back to [19,20,22,23] for the case of $A_{\infty}$-structures. Before stating it, we need to introduce some notation on rooted trees.

Let $T_{k}$ (respectively $P T_{k}$ ) be the set of isomorphism classes of directed rooted trees (respectively planar rooted trees) with internal vertices of valence at least two and exactly $k$ leaves. Let $\left(C,\left\{\Delta_{k}\right\}\right)$ be an $A_{\infty}$-coalgebra and let $(C, V, i, p, h)$ be a homotopy retract of $C$. For each planar tree $T$ in $P T_{k}$, we define a linear map $\Delta_{T}: V \rightarrow V^{\otimes k}$ as follows. The leaves of the tree are labeled by $p$, each internal edge is labelled by $h$ and the root edge is labelled by $i$. Every internal vertex $v$ is labelled by the operation $\Delta_{r}$, where $r$ is the number of input edges of $v$. Moving up from the root to the leaves one defines $\Delta_{T}$ as the composition of the different labels. For example, the tree $T$

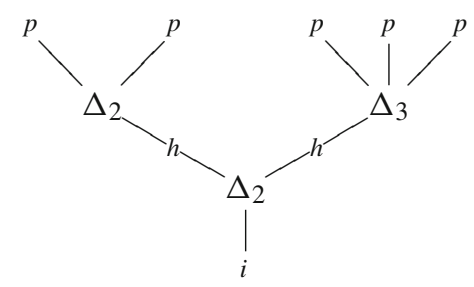

yields the map $\Delta_{T}=\left(\left((p \otimes p) \circ \Delta_{2} \circ h\right) \otimes\left((p \otimes p \otimes p) \circ \Delta_{3} \circ h\right)\right) \circ \Delta_{2} \circ i$.

Similarly, if $\left(L,\left\{\ell_{k}\right\}\right)$ is an $L_{\infty}$-algebra and $(L, V, i, p, h)$ be a homotopy retract of $L$, then each tree $T$ in $T_{k}$ gives rise to a linear map $\ell_{T}: \Lambda^{k} V \rightarrow V$ in the following way. Let $\widetilde{T}$ be a planar embedding of $T$. If we label the leaves of the tree by $i$, each internal edge by $h$, the root edge by $p$ and each internal vertex by $\ell_{k}$, where $k$ is the number of input edges, then this planar embedding defines a linear map

$$
\ell_{\widetilde{T}}: V^{\otimes k} \longrightarrow V
$$

by moving down from the leaves to the root, according to the usual operadic rules. For example, for the same tree as before, the labeling reads

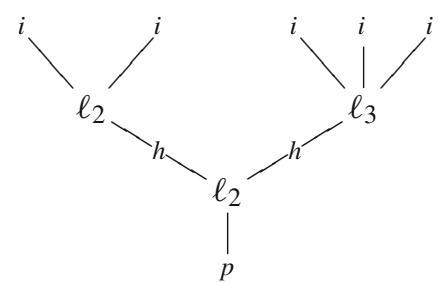

and the linear map $\ell_{\widetilde{T}}$ corresponds to

$$
p \circ \ell_{2} \circ\left(\left(h \circ \ell_{2} \circ(i \otimes i)\right) \otimes\left(h \circ \ell_{3} \circ(i \otimes i \otimes i)\right)\right) .
$$


Then, we define $\ell_{T}$ as the composition of $\ell_{\widetilde{T}}$ with the symmetrization map $\Lambda^{k} V \rightarrow$ $V^{\otimes k}$ given by

$$
v_{1} \wedge \cdots \wedge v_{k} \mapsto \sum_{\sigma \in S_{k}} \epsilon_{\sigma} \epsilon v_{\sigma(1)} \otimes \cdots \otimes v_{\sigma(n)}
$$

where $S_{k}$ denotes the symmetric group on $k$ letters, $\epsilon_{\sigma}$ denotes the signature of the permutation and $\epsilon$ stands for the sign given by the Koszul convention.

Part (i) of the following theorem follows from the tensor trick of [20] (see also [2, Section 2] for details). The second part is [28, Theorem 10.3.9].

Theorem 2.3 (Homotopy transfer theorem) Let $\left(A, d_{A}\right)$ and $\left(V, d_{V}\right)$ be two complexes and let $(A, V, i, p, h)$ be a homotopy retract. Then the following hold:

(i) If $A=\left(C,\left\{\Delta_{k}\right\}\right)$ is an $A_{\infty}$-coalgebra, then there exists an $A_{\infty}$-coalgebra structure $\left\{\Delta_{k}^{\prime}\right\}$ on $V$ and an $A_{\infty}$-quasi-isomorphism

$$
i_{\infty}:\left(V,\left\{\Delta_{k}^{\prime}\right\}\right) \longrightarrow\left(C,\left\{\Delta_{k}\right\}\right)
$$

such that $\Delta_{1}^{\prime}=d_{V}$ and $i_{\infty}^{(1)}=i$. Moreover, the transferred higher comultiplications can be explicitly described by the formula

$$
\Delta_{k}^{\prime}=\sum_{T \in P T_{k}} \Delta_{T}
$$

(ii) If $A=\left(L,\left\{\ell_{k}\right\}\right)$ is an $L_{\infty}$-algebra, then there exists an $L_{\infty}$-algebra structure $\left\{\ell_{k}^{\prime}\right\}$ on $V$ and an $L_{\infty}$-quasi-isomorphism

$$
i_{\infty}:\left(V,\left\{\ell_{k}^{\prime}\right\}\right) \longrightarrow\left(L,\left\{\ell_{k}\right\}\right)
$$

such that $\ell_{1}^{\prime}=d_{V}$ and $i_{\infty}^{(1)}=i$. Moreover, the transferred higher brackets can be explicitly described by the formula

$$
\ell_{k}^{\prime}=\sum_{T \in T_{k}} \frac{\ell_{T}}{|\operatorname{Aut}(T)|}
$$

where $\operatorname{Aut}(T)$ is the automorphism group of the tree $T$.

\subsection{Rational models for mapping spaces}

In [33] Sullivan associated to each nilpotent space $Z$ a commutative differential graded algebra $A_{P L}(Z)$. In fact, there is an adjoint pair

$$
A_{P L}: \text { sSets }^{\text {op }} \longrightarrow \text { CDGA }:\langle-\rangle,
$$


where CDGA is the category of commutative differential graded algebras, sSets is the category of simplicial sets, and $\langle-\rangle$ denotes the simplicial realization. The minimal model of $Z$ is defined as the minimal model $(\Lambda V, d)$ of $A_{P L}(Z)$. A model of $Z$ is a graded commutative differential algebra quasi-isomorphic to its minimal model. For more details, we refer to $[15,33]$.

By a model of a not necessarily connected space $Z$, such that all its components are nilpotent (or a map between them), we mean a $\mathbb{Z}$-graded commutative differential graded algebra (or a morphism) whose simplicial realization, in the sense of [33], has the same homotopy type as the singular simplicial approximation of $Z_{\mathbb{Q}}$. Similarly, by an $L_{\infty}$-model of a space $Z$ as above, we mean an $L_{\infty}$-algebra $L$ such that $\mathcal{C}^{*}(L)$ is a commutative differential graded algebra model of $Z$.

Proposition 2.4 ([3,4,8]) Let $L$ be an $L_{\infty}$-algebra and $z \in L_{-1}$ a Maurer-Cartan element. Then there is a homotopy equivalence

$$
\left\langle\mathcal{C}^{*}\left(L^{(z)}\right)\right\rangle \stackrel{\simeq}{\longrightarrow}\left\langle\mathcal{C}^{*}(L)\right\rangle_{z}
$$

where $\left\langle\mathcal{C}^{*}(L)\right\rangle_{z}$ denotes the connected component containing the 0 -simplex associated to $z$.

Note that this generalizes the notion of differential graded Lie model of a finite type nilpotent space $Z$, since in this case $\mathcal{C}^{*}$ coincides with the classical cochain functor and the only Maurer-Cartan element in $L$ is the zero element.

Similarly, we say that an $A_{\infty}$-coalgebra model of $Z$ is a cocommutative $A_{\infty}$-coalgebra $C$ such that $\mathcal{L}(C)$ is a differential graded Lie model of $Z$, where $\mathcal{L}$ denotes the generalized Quillen functor; see [8] for further details. This functor assigns to a cocommutative $A_{\infty}$-coalgebra $C$ an induced differential graded Lie algebra structure on $\mathbb{L}\left(s^{-1} C\right)$ whose differential $\partial=\sum_{k \geq 1} \partial_{k}$ with $\partial_{k}: s^{-1} C \rightarrow \mathbb{L}^{k}\left(s^{-1} C\right)$ is determined by $\Delta_{k}$ in the same way as the classical Quillen functor assigns a differential $\partial=\partial_{1}+\partial_{2}$ on $\mathbb{L}\left(s^{-1} C\right)$; see, e.g., [15, IV.22]. In fact, if $C$ is a cocommutative differential graded coalgebra viewed as a cocommutative $A_{\infty}$-coalgebra, then $\mathcal{L}$ coincides with the classical Quillen functor.

In the rest of the paper $X$ will always denote a nilpotent finite $C W$-complex and $Y$ will always denote a rational finite type $C W$-complex, although most of the results can be stated if we remove the finiteness assumption on $X$, as in $[9,10]$.

We recall briefly the Haefliger model [21] of the mapping space via the functorial description of Brown-Szczarba [4]. Let $B$ be a finite dimensional differential graded algebra model of $X$ and let $(\Lambda V, d)$ be a Sullivan (non-necessarily minimal) model of $Y$. We denote by $B^{\sharp}$ the differential coalgebra dual of $B$ with the grading $\left(B^{\sharp}\right)^{-n}=B_{n}^{\sharp}=\operatorname{Hom}\left(B^{n}, \mathbb{Q}\right)$ and consider the free commutative algebra $\Lambda\left(\Lambda^{+} V \otimes B^{\sharp}\right)$ generated by the $\mathbb{Z}$-graded vector space $\Lambda^{+} V \otimes B^{\sharp}$, endowed with the differential $d$ induced by the ones on $(\Lambda V, d)$ and on $\left(B^{\sharp}, \delta\right)$. Let $J$ be the differential ideal generated by $1 \otimes 1-1$, and the elements of the form

$$
v_{1} v_{2} \otimes \beta-\sum_{j}(-1)^{\left|v_{2}\right|\left|\beta_{j}^{\prime}\right|}\left(v_{1} \otimes \beta_{j}^{\prime}\right)\left(v_{2} \otimes \beta_{j}^{\prime \prime}\right), \quad v_{1}, v_{2} \in V,
$$


where $\Delta \beta=\sum_{j} \beta_{j}^{\prime} \otimes \beta_{j}^{\prime \prime}$. The inclusion $V \otimes B^{\sharp} \hookrightarrow \Lambda^{+} V \otimes B^{\sharp}$ induces an isomorphism of graded algebras

$$
\rho: \Lambda\left(V \otimes B^{\sharp}\right) \stackrel{\cong}{\longrightarrow} \Lambda\left(\Lambda^{+} V \otimes B^{\sharp}\right) / J,
$$

and thus $\widetilde{d}=\rho^{-1} d \rho$ defines a differential in $\Lambda\left(V \otimes B^{\sharp}\right)$ and the following holds:

Theorem 2.5 ([4,21]) The commutative differential graded algebra $\left(\Lambda\left(V \otimes B^{\sharp}\right), \widetilde{d}\right)$ is a model of $\operatorname{map}(X, Y)$, and the commutative differential graded algebra $(\Lambda(V \otimes$ $\left.\left.B_{+}^{\sharp}\right), \widetilde{d}\right)$ is a model of $\operatorname{map}^{*}(X, Y)$.

Now write $B^{\sharp}=A \oplus \delta A \oplus H$, where $H \cong H\left(B^{\sharp}\right)$, with basis $\left\{a_{j}\right\},\left\{b_{j}\right\}$ and $\left\{h_{s}\right\}$. Thus $\delta a_{j}=b_{j}$ and $\delta h_{s}=0$. Additionally, since $(\Lambda V, d)$ is a Sullivan algebra, we can choose a basis $\left\{v_{i}\right\}$ for $V$ for which $d v_{i} \in \Lambda V_{<i}$. Then we have:

Lemma 2.6 ([4,5]) The commutative differential graded algebra $\left(\Lambda\left(V \otimes B^{\sharp}\right), \widetilde{d}\right)$ splits as $(\Lambda W, \widetilde{d}) \otimes \Lambda(U \oplus \widetilde{d} U)$, where

(i) $U$ is generated by $u_{i j}=v_{i} \otimes a_{j}$;

(ii) $W$ is generated by $w_{i s}=v_{i} \otimes h_{s}-x_{i s}$, for suitable $x_{i s} \in \Lambda\left(V_{<i} \otimes B^{\sharp}\right)$;

(iii) $\widetilde{d} w_{i s} \in \Lambda\left\{w_{m s}\right\}_{m<i}$;

(iv) if $\widetilde{d}\left(v_{i} \otimes h_{s}\right)$ is decomposable, so is $\widetilde{d} w_{i s}$.

Proof We proceed by induction on $i$. Suppose that $w_{m s}$ has been defined for $m<i$ satisfying the lemma for $\left(\Lambda\left(V_{<i} \otimes B^{\sharp}\right), \widetilde{d}\right)$. Now, since $\widetilde{d}\left(v_{i} \otimes h_{s}\right)=\rho^{-1}\left[d v_{i} \otimes h_{s}\right]$ belongs to $\Lambda\left(V_{<i} \otimes B^{\sharp}\right)$, and

$$
\Lambda\left(V_{<i} \otimes B^{\sharp}\right)=\Lambda\left\{w_{m s}\right\}_{m<i} \otimes \Lambda\left\{u_{m j}, \widetilde{d} u_{m j}\right\}_{m<i},
$$

we can write $\widetilde{d}\left(v_{i} \otimes h_{s}\right)=\Gamma_{1}+\Gamma_{2}$, where

$$
\Gamma_{1} \in \Lambda\left\{w_{m s}\right\}_{m<i} \text { and } \Gamma_{2} \in \Lambda\left\{w_{m s}\right\}_{m<i} \otimes \Lambda^{+}\left\{u_{m j}, \widetilde{d} u_{m j}\right\}_{m<i}
$$

The ideal $\Lambda\left\{w_{m s}\right\}_{m<i} \otimes \Lambda^{+}\left\{u_{m j}, \widetilde{d} u_{m j}\right\}_{m<i}$ is acyclic, since by inductive hypothesis $\Lambda\left\{w_{m s}\right\}_{m<i}$ is $\widetilde{d}$-stable. Therefore, $\Gamma_{2}$ is a boundary, i.e., $\Gamma_{2}=\widetilde{d} x_{i s}$ for some $x_{i s} \in$ $\Lambda\left\{w_{m s}\right\}_{m<i} \otimes \Lambda^{+}\left\{u_{m j}, \widetilde{d} u_{m j}\right\}_{m<i}$. We define $w_{i s}=v_{i} \otimes h_{s}-x_{i s}$, which clearly satisfies (ii), (iii) and (iv). To finish, observe that $\widetilde{d} u_{i j}=\widetilde{d}\left(v_{i} \otimes a_{j}\right)= \pm v_{i} \otimes b_{j}+$ $\rho^{-1}\left[\left(d v_{i}\right) \otimes a_{j}\right]$, where $\rho^{-1}\left[\left(d v_{i}\right) \otimes a_{j}\right] \in \Lambda\left(V_{<i} \otimes B^{\sharp}\right)$. Hence,

$$
\Lambda\left(V_{\leq i} \otimes B^{\sharp}\right)=\Lambda\left\{w_{m s}\right\}_{m \leq i} \otimes \Lambda\left\{u_{m j}, \tilde{d} u_{m j}\right\}_{m \leq i},
$$

and this completes the proof.

We can endow the free algebra $\Lambda(V \otimes H)$ with a differential $\widehat{d}$ so that the map

$$
\sigma:(\Lambda(V \otimes H), \widehat{d}) \stackrel{\cong}{\longrightarrow}(\Lambda W, \widetilde{d})
$$


is an isomorphism of differential graded algebras, and therefore $(\Lambda(V \otimes H), \widehat{d})$ is a model of $\operatorname{map}(X, Y)$ called the reduced Brown-Szczarba model. The differential $\widehat{d}$ of this model can be easily described. The case $\left(\Lambda V, d=d_{1}+d_{2}\right)$ is proved in [5, Lemma 2.8] and the same proof also works in the general case.

Consider the composition $\theta=\sigma^{-1} p: \Lambda\left(V \otimes B^{\sharp}\right)=\Lambda W \otimes \Lambda(U \oplus \widetilde{d} U) \rightarrow$ $\Lambda(V \otimes H)$, where $p: \Lambda W \otimes \Lambda(U \oplus \widetilde{d} U) \stackrel{\simeq}{\rightarrow} \Lambda W$ is the projection.

Lemma 2.7 ([5]) The morphism $\theta$ operates on the generators as follows:

$\theta(v \otimes b)= \begin{cases}v \otimes b & \text { if } b \in H, \\ 0 & \text { if } b \in A, \\ (-1)^{|v|+1} \sum_{k} \sum_{i, j} \varepsilon \theta\left(v_{i}^{(1)} \otimes z_{j}^{(1)}\right) \cdots \theta\left(v_{i}^{(k)} \otimes z_{j}^{(k)}\right) & \text { if } b \in \delta A,\end{cases}$

where in the last case $b=\delta a, \Delta^{(k-1)} a=\sum_{j} z_{j}^{(1)} \otimes \cdots \otimes z_{j}^{(k)}, d_{k} v=\sum_{i} v_{i}^{(1)} \cdots v_{i}^{(k)}$, and $\varepsilon$ is the sign given by Koszul convention.

Lemma 2.8 ([5]) The differential in the free commutative differential graded algebra $(\Lambda(V \otimes H), \widehat{d})$ is given by the formula:

$$
\widehat{d}(v \otimes h)=d_{1} v \otimes h+\sum_{k} \sum_{i, j} \varepsilon \theta\left(v_{i}^{(1)} \otimes z_{j}^{(1)}\right) \cdots \theta\left(v_{i}^{(k)} \otimes z_{j}^{(k)}\right),
$$

where $\Delta^{(k-1)} h=\sum_{j} z_{j}^{(1)} \otimes \cdots \otimes z_{j}^{(k)}$ and $d_{k} v=\sum_{i} v_{i}^{(1)} \cdots v_{i}^{(k)}, k \geq 2$.

We can describe a formula for $\widehat{d}_{j}$ in terms of directed rooted trees. Let $T \in T_{j}$ be a directed rooted tree with $j$ leaves. We can associate to $T$ two different linear maps $T_{V}: V \rightarrow V^{\otimes j}$ and $T_{H}: H \rightarrow H^{\otimes j}$ moving up from the root to the leaves. In the first case we label each internal vertex with $r$ input edges with $\mathcal{S} \circ d_{r}$, where $\mathcal{S}: \Lambda^{r} V \rightarrow V^{\otimes r}$ is the symmetrization map. In the second case we label the root with $i$, each internal vertex with $r$ input edges with $\Delta^{(r-1)}$, each internal edge with $k$ and each leaf with $p$, where $i, p$ and $k$ are described in the homotopy retract (3.1), taking $C=B^{\sharp}$. Here is an example of the previous labeling defining $T_{V}$ and $T_{H}$ respectively, for a concrete tree:
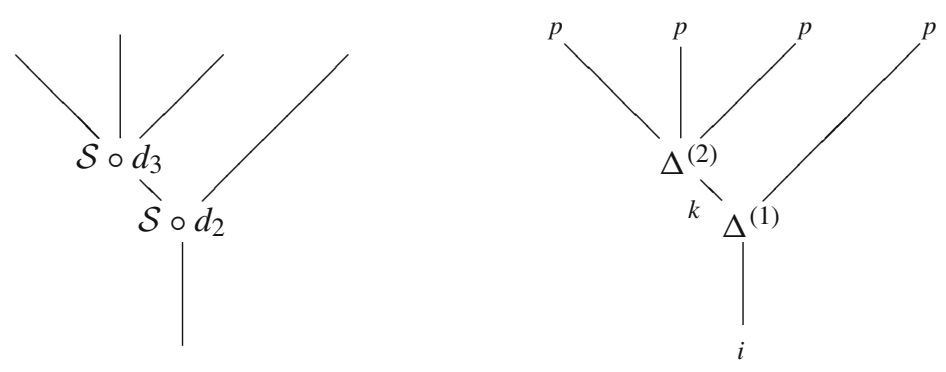

Now we define a linear map

$$
\Theta_{j}: V^{\otimes j} \times H^{\otimes j} \rightarrow \Lambda^{j}(V \otimes H),
$$


by $\Theta_{j}\left(v_{1} \otimes \cdots \otimes v_{j}, h_{1} \otimes \cdots \otimes h_{j}\right)=\varepsilon\left(v_{1} \otimes h_{1}\right) \cdots\left(v_{j} \otimes h_{j}\right)$, where $\varepsilon$ is the sign given by the Koszul convention.

Using the formula described in Lemma 2.8, we can check the following:

Lemma 2.9 The jth part of the differential in the free commutative differential graded algebra $(\Lambda(V \otimes H), \widehat{d})$ is given by the formula:

$$
\widehat{d}_{j}(v \otimes h)=\sum_{T \in T_{j}} \frac{1}{|\operatorname{Aut}(T)|} \Theta_{j}\left(T_{V}(v), T_{H}(h)\right)
$$

If $C$ is a coalgebra model of $X$ and $L$ is an $L_{\infty}$-model of $Y$, then we can endow the complex $\operatorname{Hom}(C, L)$ with an $L_{\infty}$-algebra structure modeling the space of continuous functions from $X$ to $Y$. More concretely,

Theorem 2.10 ([3,9]) The complex of linear maps $\operatorname{Hom}(C, L)$ with brackets

$$
\begin{aligned}
& \ell_{1}(f)=\ell_{1} \circ f+(-1)^{|f|+1} f \circ \delta, \\
& \ell_{k}\left(f_{1}, \ldots, f_{k}\right)=\left[-, .{ }^{(k)} .,-\right]_{L} \circ\left(f_{1} \otimes \cdots \otimes f_{k}\right) \circ \Delta^{(k-1)}, k \geq 2,
\end{aligned}
$$

is an $L_{\infty}$-algebra modeling $\operatorname{map}(X, Y)$.

Similarly, the complex $\operatorname{Hom}(\bar{C}, L)$, where $\bar{C}=\operatorname{ker} \varepsilon$ is the kernel of the augmentation $\varepsilon: C \rightarrow \mathbb{Q}$, with the same brackets replacing $\Delta$ with $\bar{\Delta}$, is an $L_{\infty}$-model for map* $(X, Y)$, the space of based functions.

\section{An explicit $L_{\infty}$-structure on $\operatorname{Hom}\left(H_{*}(X, \mathbb{Q}), \pi_{*}(\Omega Y) \otimes \mathbb{Q}\right)$}

In this section, we describe an explicit $L_{\infty}$-structure on the complex of linear maps $\operatorname{Hom}(H, L)$, where $L$ is an $L_{\infty}$-algebra and $H$ is the homology of a coalgebra $C$ with the transferred $A_{\infty}$-structure.

If $C$ is a cocommutative differential graded coalgebra and $H \cong H(C)$ denotes the homology of $C$, then the transferred $A_{\infty}$-coalgebra structure on $H$, whose higher comultiplications are called higher Massey coproducts (cf. [28, 10.3.12]) is described as follows. We can decompose $(C, \delta, \Delta)$ as $A \oplus \delta A \oplus H$ with basis $\left\{a_{j}\right\},\left\{\delta a_{j}\right\}$ and $\left\{h_{s}\right\}$. Thus, $\delta=0$ in $H$ and $\delta: A \rightarrow \delta A$ is an isomorphism. This decomposition induces a homotopy retract

$$
k \bigodot_{T}(C, \delta) \underset{r_{i}}{\stackrel{p}{\gtrless}}(H, 0)
$$

given by $p\left(a_{j}\right)=p\left(\delta a_{j}\right)=0, p\left(h_{s}\right)=h_{s} ; i\left(h_{s}\right)=h_{s} ; k\left(a_{j}\right)=k\left(h_{s}\right)=0$ and $k\left(\delta a_{j}\right)=a_{j}$. Then by Theorem 2.3(i), we can transfer the cocommutative differential graded coalgebra structure on $C$ to an $A_{\infty}$-coalgebra structure on $H$. For example, 
since $C$ has no higher order coproducts, the operation $\Delta_{3}^{\prime}$ on $H$ given by the formula of Theorem 2.3(i) is provided by the trees
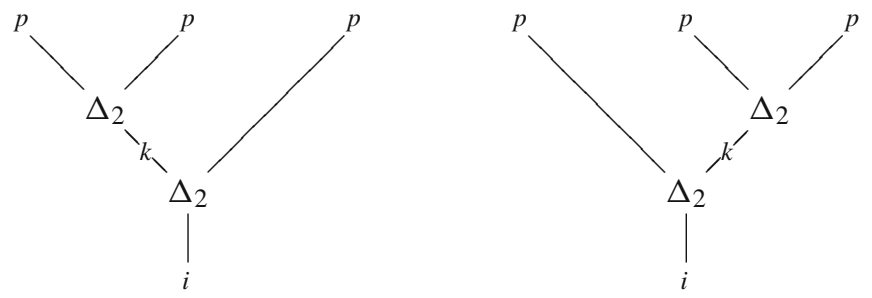

Explicitly,

$$
\begin{aligned}
\Delta_{3}^{\prime}(h)= & (p \otimes p \otimes \mathrm{id}) \circ\left(\Delta_{2} \otimes \mathrm{id}\right) \circ(k \otimes p) \circ \Delta_{2} \circ i(h) \\
& \pm(\mathrm{id} \otimes p \otimes p) \circ\left(\mathrm{id} \otimes \Delta_{2}\right) \circ(p \otimes k) \circ \Delta_{2} \circ i(h) \\
= & \sum_{j}(p \otimes p \otimes \mathrm{id}) \circ\left(\Delta_{2} \otimes \mathrm{id}\right) \circ(k \otimes p)\left(z_{j}^{\prime} \otimes z_{j}^{\prime \prime}\right) \\
& \pm \sum_{j}(\mathrm{id} \otimes p \otimes p) \circ\left(\mathrm{id} \otimes \Delta_{2}\right) \circ(p \otimes k)\left(z_{j}^{\prime} \otimes z_{j}^{\prime \prime}\right),
\end{aligned}
$$

where $\Delta_{2}(h)=\sum_{j} z_{j}^{\prime} \otimes z_{j}^{\prime \prime}$. For a term of the form $z_{j}^{\prime} \otimes z_{j}^{\prime \prime}=\delta a \otimes h^{\prime}$, the $j$ th term in the above summation is

$$
\begin{aligned}
& (p \otimes p \otimes \mathrm{id}) \circ\left(\Delta_{2} \otimes \mathrm{id}\right) \circ\left(k(\delta a) \otimes p\left(h^{\prime}\right)\right) \\
& \quad \pm(\mathrm{id} \otimes p \otimes p)\left(\mathrm{id} \otimes \Delta_{2}\right)\left(p(\delta a) \otimes k\left(h^{\prime}\right)\right) \\
& =(p \otimes p \otimes \mathrm{id}) \circ\left(\Delta_{2} \otimes \mathrm{id}\right) \circ\left(a \otimes h^{\prime}\right) \\
& =\sum_{i}(p \otimes p \otimes \mathrm{id}) \circ\left(x_{i}^{\prime} \otimes x_{i}^{\prime \prime} \otimes h^{\prime}\right) \\
& =\sum_{i} p\left(x_{i}^{\prime}\right) \otimes p\left(x_{i}^{\prime \prime}\right) \otimes h^{\prime},
\end{aligned}
$$

where $\Delta_{2}(a)=\sum_{i} x_{i}^{\prime} \otimes x_{i}^{\prime \prime}$.

Replacing $C$ by the kernel of the augmentation $\bar{C}$ and using the decomposition $\bar{C}=A \oplus \delta A \oplus \bar{H}$, we can proceed similarly as above to obtain a transferred $A_{\infty^{-}}$ coalgebra structure on $\bar{H}$. The following result relates the Quillen minimal model of a differential graded Lie algebra with the above higher Massey coproducts:

Theorem 3.1 The transferred $A_{\infty}$-coalgebra structure on $\bar{H}$ is cocommutative and $\mathcal{L}(\bar{H})$ is the Quillen minimal model of $\mathcal{L}(\bar{C})$.

Proof Since the kernel of the augmentation $\bar{C}$ is cocommutative, the transferred $A_{\infty}$ coalgebra structure on $\bar{H}$ is also cocommutative (cf. [13, Theorem 12]). Then we can apply the Quillen functor to diagram (3.1) and we get a quasi-isomorphism 
$\left(\mathbb{L}\left(s^{-1} \bar{H}\right), \partial\right) \stackrel{\sim}{\rightarrow}(\mathcal{L}(\bar{C}), \partial)$. The $k$ th part $\partial_{k}$ of the differential in $\left(\mathbb{L}\left(s^{-1} \bar{H}\right), \partial\right)$ is determined by the higher Massey coproduct $\bar{\Delta}_{k}^{\prime}$ in $\bar{H}$.

Moreover, by the computations of $\bar{\Delta}_{k}^{\prime}$ made above, if we start by decomposing $(\bar{C}, \delta, \bar{\Delta})$ as $A \oplus \delta A \oplus \bar{H}$, where $\bar{H} \cong H(\bar{C})$ with basis $\left\{a_{j}\right\},\left\{\delta a_{j}\right\}$ and $\left\{h_{s}\right\}$, then we can easily check that the differential on $\mathbb{L}\left(s^{-1} \bar{H}\right)$ is described by

$$
\partial s^{-1} h=\frac{1}{2} \sum_{j}(-1)^{\left|z_{j}^{\prime}\right|}\left[\lambda\left(z_{j}^{\prime}\right), \lambda\left(z_{j}^{\prime \prime}\right)\right]
$$

where $\bar{\Delta} h=\sum_{j} z_{j}^{\prime} \otimes z_{j}^{\prime \prime}$ and

$$
\lambda(h)=s^{-1} h, \quad \lambda(a)=0, \quad \lambda(\delta a)=\frac{1}{2} \sum_{i}(-1)^{\left|x_{i}^{\prime}\right|}\left[\lambda\left(x_{i}^{\prime}\right), \lambda\left(x_{i}^{\prime \prime}\right)\right],
$$

where $\bar{\Delta} a=\sum_{i} x_{i}^{\prime} \otimes x_{i}^{\prime \prime}$.

Finally, by [5, Theorem 2.1] or [15, Theorem 22.13], $\left(\mathbb{L}\left(s^{-1} \bar{H}\right), \partial\right)$ agrees with the Quillen minimal model of $(\mathcal{L}(\bar{C}), \partial)$.

The following is the main result of this article:

Theorem 3.2 Let $C$ be a finite dimensional cocommutative differential graded coalgebra model of a finite nilpotent $C W$-complex $X$, and let $L$ be an $L_{\infty}$-model of a rational $C W$-complex of finite type $Y$.

(i) There is an explicit $L_{\infty}$-structure in $\operatorname{Hom}(H, L)$, where $H$ denotes the homology of $C$.

(ii) With the above structure $\mathcal{C}^{*}(\operatorname{Hom}(H, L)) \cong(\Lambda(V \otimes H), \widehat{d})$, the reduced Brown$S z c z a r b a$ model of $\operatorname{map}(X, Y)$, where $V=(s L)^{\sharp}$. Hence $\operatorname{Hom}(H, L)$ is an $L_{\infty}-$ model for the mapping space.

If we replace $H$ by the homology $\bar{H}$ of the kernel of the augmentation of $C$, then we get the same results for the pointed mapping space map* $(X, Y)$.

Proof The homotopy retract (3.1) induces a new homotopy retract

$$
k^{*} \bigcirc(\operatorname{Hom}(C, L), \delta) \underset{p^{*}}{\stackrel{i^{*}}{\gtrless}}(\operatorname{Hom}(H, L), \delta),
$$

where both complexes of linear maps have the usual differentials. Since $i$ and $p$ are quasi-isomorphism so are $i^{*}$ and $p^{*}$. Observe that $\operatorname{Hom}(C, L)$ can be endowed with a natural $L_{\infty}$-algebra structure modeling mapping spaces under suitable conditions [3, 9]. Hence we can apply Theorem 2.3(ii) to obtain an $L_{\infty}$-structure $\left\{\ell_{k}^{\prime}\right\}$ on $\operatorname{Hom}(H, L)$. This proves part (i).

In order to prove (ii), it is enough to see that Eq. (2.1) holds, which in this case amounts to check that

$$
\left\langle\widehat{d}_{j}(v \otimes h) ; s f_{1} \wedge \cdots \wedge s f_{j}\right\rangle=(-1)^{\epsilon}\left\langle v \otimes h ; s \ell_{j}^{\prime}\left(f_{1}, \ldots, f_{j}\right)\right\rangle .
$$


In what follows we work modulo signs and summations where necessary in order to simplify the computations. In view of the isomorphism $V \otimes H \cong(s \operatorname{Hom}(H, L))^{\sharp}$, and Theorem 2.3(ii), the right hand part of the above equation can be written as:

$$
\begin{aligned}
\left\langle v \otimes h ; s \ell_{j}^{\prime}\left(f_{1}, \ldots, f_{j}\right)\right\rangle & = \pm\left\langle v ; s \ell_{j}^{\prime}\left(f_{1}, \ldots, f_{j}\right)(h)\right\rangle \\
& = \pm \sum_{T \in T_{j}} \frac{1}{|\operatorname{Aut}(T)|}\left\langle v ; s \ell_{T}\left(f_{1}, \ldots, f_{j}\right)(h)\right\rangle \\
& =\sum_{T \in T_{j}}\left\langle\frac{1}{|\operatorname{Aut}(T)|} \Theta_{j}\left(T_{V}(v), T_{H}(h)\right) ; s f_{1} \wedge \cdots \wedge s f_{j}\right\rangle,
\end{aligned}
$$

where we have used in the last equality Eq. (2.1). The result is now a consequence of Lemma 2.9. The same proof works for the pointed case.

Example 3.3 In this example, we describe explicitly $\ell_{j}^{\prime}$ in the case $j=3$. In what follows we have omitted the suspensions in order to simplify the notation. The explicit formula for $\ell_{3}^{\prime}$ is provided by the trees
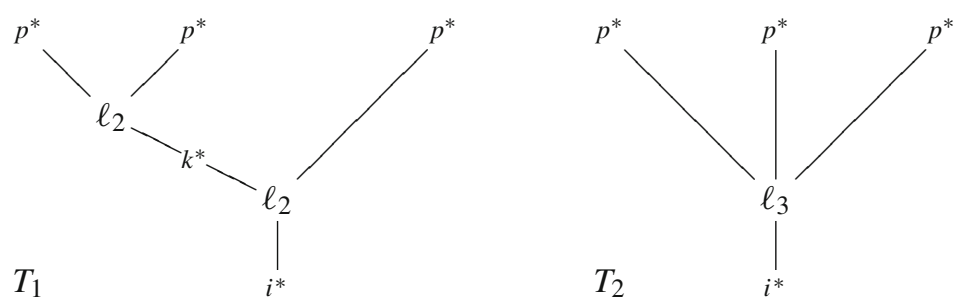

Therefore, if $f_{1}, f_{2}$ and $f_{3}$ are elements of $\operatorname{Hom}(H, L)$ and $h$ is an element of $H$, then $\ell_{3}^{\prime}\left(f_{1}, f_{2}, f_{3}\right)(h)$ is expressed in terms of the maps

$$
\begin{aligned}
\ell_{\widetilde{T}_{2}}\left(f_{1}, f_{2}, f_{3}\right)(h) & =i_{*} \ell_{3}\left(p^{*} f_{1}, p^{*} f_{2}, p^{*} f_{3}\right)(h) \\
& =[-,-,-]_{L} \circ\left(p^{*} f_{1} \otimes p^{*} f_{2} \otimes p^{*} f_{3}\right) \circ \Delta^{(2)}(h) \\
& =\sum_{j}(-1)^{\left|z_{j}^{\prime}\right|\left(\left|f_{2}\right|+\left|f_{3}\right|\right)+\left|z_{j}^{\prime \prime}\right|\left|f_{3}\right|}\left[f_{1} p\left(z_{j}^{\prime}\right), f_{2} p\left(z_{j}^{\prime \prime}\right), f_{3} p\left(z_{j}^{\prime \prime \prime}\right)\right]_{L},
\end{aligned}
$$

where $\Delta^{(2)}(h)=(\Delta \otimes \mathrm{id}) \circ \Delta(h)=\sum_{j} z_{j}^{\prime} \otimes z_{j}^{\prime \prime} \otimes z_{j}^{\prime \prime \prime}$, and

$$
\begin{aligned}
\ell_{\widetilde{T}_{1}}\left(f_{1}, f_{2}, f_{3}\right)(h) & =i^{*} \ell_{2}\left(k^{*} \ell_{2}\left(p^{*} f_{1}, p^{*} f_{2}\right), p^{*} f_{3}\right)(h) \\
& =[-,-]_{L} \circ\left(k^{*} \ell_{2}\left(p^{*} f_{1}, p^{*} f_{2}\right) \otimes p^{*} f_{3}\right) \circ \Delta(h) \\
& =\sum_{j}(-1)^{\left|z_{j}^{\prime}\right|\left|f_{3}\right|}\left[\ell_{2}\left(p^{*} f_{1}, p^{*} f_{2}\right) \circ k\left(z_{j}^{\prime}\right), f_{3} p\left(z_{j}^{\prime \prime}\right)\right]_{L},
\end{aligned}
$$

where $\Delta(h)=\sum_{j} z_{j}^{\prime} \otimes z_{j}^{\prime \prime}$. For a term of the form $z_{j}^{\prime} \otimes z_{j}^{\prime \prime}=\delta a \otimes h^{\prime}$ the $j$ th term in the above summation equals 


$$
\sum_{i}(-1)^{(|a|+1)\left|f_{3}\right|+\left|x_{i}^{\prime}\right|\left|f_{2}\right|}\left[\left[f_{1} p\left(x_{i}^{\prime}\right), f_{2} p\left(x_{i}^{\prime \prime}\right)\right]_{L}, f_{3} h^{\prime}\right]_{L},
$$

where $\Delta(a)=\sum_{i} x_{i}^{\prime} \otimes x_{i}^{\prime \prime}$.

We also make explicit the computations to check that

$$
\left\langle v \otimes h ; \ell_{3}^{\prime}\left(f_{1}, f_{2}, f_{3}\right)\right\rangle= \pm\left\langle\widehat{d}_{3}(v \otimes h) ; f_{1} \wedge f_{2} \wedge f_{3}\right\rangle .
$$

In order to simplify these computations, in what follows we set $\Delta(h)=\delta a \otimes h^{\prime} \pm h^{\prime} \otimes \delta a$ and $\Delta(a)=x^{\prime} \otimes x^{\prime \prime} \pm x^{\prime \prime} \otimes x^{\prime}$.

First we compute $\widehat{d}_{3}(v \otimes h)$ :

$$
\begin{aligned}
\left(T_{1}\right)_{V}(v)= & \left(\left(\mathcal{S} \circ d_{2}\right) \otimes \mathrm{id}\right) \circ\left(\mathcal{S} \circ d_{2}\right)(v)=\left(\left(\mathcal{S} \circ d_{2}\right) \otimes \mathrm{id}\right)(u \otimes w \pm w \otimes u) \\
= & \left(u^{\prime} \otimes u^{\prime \prime} \otimes w\right) \pm\left(u^{\prime \prime} \otimes u^{\prime} \otimes w\right) \pm\left(w^{\prime} \otimes w^{\prime \prime} \otimes u\right) \pm\left(w^{\prime \prime} \otimes w^{\prime} \otimes u\right) . \\
\left(T_{2}\right)_{V}(v)= & \left(\mathcal{S} \circ d_{3}\right)(v) \\
= & (p \otimes q \otimes r) \pm(p \otimes r \otimes q) \pm(q \otimes p \otimes r) \pm(q \otimes r \otimes p) \\
& \pm(r \otimes p \otimes q) \pm(r \otimes q \otimes p) .
\end{aligned}
$$

$\left.\left(T_{1}\right)_{H}(h)=(p \otimes p \otimes \mathrm{id}) \circ\left(\Delta^{(1)} \circ k\right) \otimes p\right)\left(\delta a \otimes h^{\prime} \pm h^{\prime} \otimes \delta a\right)$

$=(p \otimes p \otimes \mathrm{id})\left(\Delta^{(1)}(a) \otimes h^{\prime}\right)=p\left(x^{\prime}\right) \otimes p\left(x^{\prime \prime}\right) \otimes h^{\prime} \pm p\left(x^{\prime \prime}\right) \otimes p\left(x^{\prime}\right) \otimes h^{\prime}$.

$\left(T_{2}\right)_{H}(h)=(p \otimes p \otimes p) \circ \Delta^{(2)} \circ i(h)=\sum_{j} p\left(z_{j}^{\prime}\right) \otimes p\left(z_{j}^{\prime \prime}\right) \otimes p\left(z_{j}^{\prime \prime \prime}\right)$.

We can now apply Lemma 2.9 or Lemma 2.8 to obtain

$$
\begin{aligned}
\widehat{d}_{3}(v \otimes h)= & \frac{1}{\left|\operatorname{Aut}\left(T_{1}\right)\right|} \Theta\left(\left(T_{1}\right)_{V}(v),\left(T_{1}\right)_{H}(h)\right)+\frac{1}{\left|\operatorname{Aut}\left(T_{2}\right)\right|} \Theta\left(\left(T_{2}\right)_{V}(v),\left(T_{2}\right)_{H}(h)\right) \\
= & \left(u^{\prime} \otimes p\left(x^{\prime}\right)\right)\left(u^{\prime \prime} \otimes p\left(x^{\prime \prime}\right)\right)\left(w \otimes h^{\prime}\right) \pm\left(u^{\prime} \otimes p\left(x^{\prime \prime}\right)\right)\left(u^{\prime \prime} \otimes p\left(x^{\prime}\right)\right)\left(w \otimes h^{\prime}\right) \\
& \pm\left(u \otimes h^{\prime}\right)\left(w^{\prime} \otimes p\left(x^{\prime}\right)\right)\left(w^{\prime \prime} \otimes p\left(x^{\prime \prime}\right)\right) \pm\left(u \otimes h^{\prime}\right)\left(w^{\prime} \otimes p\left(x^{\prime \prime}\right)\right)\left(w^{\prime \prime} \otimes p\left(x^{\prime}\right)\right) \\
& \pm \sum_{j}\left(p \otimes p\left(z_{j}^{\prime}\right)\right)\left(q \otimes p\left(z_{j}^{\prime \prime}\right)\right)\left(r \otimes p\left(z_{j}^{\prime \prime \prime}\right)\right) .
\end{aligned}
$$

Then, on the one hand we have

$$
\begin{aligned}
\left\langle\widehat{d}_{3}(v \otimes h) ; f_{1} \wedge f_{2} \wedge f_{3}\right\rangle= & \sum_{\sigma \in S_{3}}\left\{\left\langle u^{\prime} ; f_{\sigma(1)}\left(p x^{\prime}\right)\right\rangle\left\langle u^{\prime \prime} ; f_{\sigma(2)}\left(p x^{\prime \prime}\right)\right\rangle\left\langle w ; f_{\sigma(3)}\left(h^{\prime}\right)\right\rangle\right. \\
& \pm\left\langle u^{\prime} ; f_{\sigma(1)} p\left(x^{\prime \prime}\right)\right\rangle\left\langle u^{\prime \prime} ; f_{\sigma(2)} p\left(x^{\prime}\right)\right\rangle\left\langle w ; f_{\sigma(3)}\left(h^{\prime}\right)\right\rangle \\
& \pm\left\langle u ; f_{\sigma(1)}\left(h^{\prime}\right)\right\rangle\left\langle w^{\prime} ; f_{\sigma(2)} p\left(x^{\prime}\right)\right\rangle\left\langle w^{\prime \prime} ; f_{\sigma(3)} p\left(x^{\prime \prime}\right)\right\rangle \\
& \left. \pm\left\langle u ; f_{\sigma(1)}\left(h^{\prime}\right)\right\rangle\left\langle w^{\prime} ; f_{\sigma(2)} p\left(x^{\prime \prime}\right)\right\rangle\left\langle w^{\prime \prime} ; f_{\sigma(3)} p\left(x^{\prime}\right)\right\rangle\right\} \\
& \pm \sum_{\sigma \in S_{3}} \sum_{j}\left\langle p ; f_{\sigma(1)} p\left(z_{j}^{\prime}\right)\right\rangle\left\langle q ; f_{\sigma(1)} p\left(z_{j}^{\prime \prime}\right)\right\rangle\left\langle r ; f_{\sigma(3)} p\left(z_{j}^{\prime \prime \prime}\right)\right\rangle \\
= & (\dagger)+(\ddagger) .
\end{aligned}
$$


And on the other hand

$$
\begin{aligned}
\left\langle v \otimes h ; \ell_{3}^{\prime}\left(f_{1}, f_{2}, f_{3}\right)\right\rangle= & \pm \frac{1}{\left|\operatorname{Aut}\left(T_{1}\right)\right|}\left\langle v ; \ell_{T_{1}}\left(f_{1}, f_{2}, f_{3}\right)(h)\right\rangle \\
& \pm \frac{1}{\left|\operatorname{Aut}\left(T_{2}\right)\right|}\left\langle v ; \ell_{T_{2}}\left(f_{1}, f_{2}, f_{3}\right)(h)\right\rangle
\end{aligned}
$$

As we have seen before,

$$
\begin{aligned}
& \ell_{\widetilde{T}_{2}}\left(f_{1}, f_{2}, f_{3}\right)(h)= \pm \sum_{j}\left[f_{1} p\left(z_{j}^{\prime}\right), f_{2} p\left(z_{j}^{\prime \prime}\right), f_{3} p\left(z_{j}^{\prime \prime \prime}\right)\right]_{L}, \\
& \ell_{\widetilde{T}_{1}}\left(f_{1}, f_{2}, f_{3}\right)(h)= \pm\left[\left[f_{1} p\left(x^{\prime}\right), f_{2} p\left(x^{\prime \prime}\right)\right]_{L}, f_{3}\left(h^{\prime}\right)\right]_{L} \pm\left[\left[f_{1} p\left(x^{\prime \prime}\right), f_{2} p\left(x^{\prime}\right)\right]_{L}, f_{3}\left(h^{\prime}\right)\right]_{L},
\end{aligned}
$$

Then, applying (2.1) to the $L_{\infty}$-algebra $L$, we have

$$
\begin{aligned}
\frac{1}{\left|\operatorname{Aut}\left(T_{2}\right)\right|}\left\langle v ; \ell_{T_{2}}\left(f_{1}, f_{2}, f_{3}\right)(h)\right\rangle & = \pm \frac{1}{3 !} \sum_{\sigma \in S_{3}} \sum_{j}\left\langle v ;\left[f_{\sigma(1)} p\left(z_{j}^{\prime}\right), f_{\sigma(2)} p\left(z_{j}^{\prime \prime}\right), f_{\sigma(3)} p\left(z_{j}^{\prime \prime \prime}\right)\right]_{L}\right\rangle \\
& = \pm \frac{1}{6} \sum_{\sigma \in S_{3}} \sum_{j}\left\langle p q r ; f_{\sigma(1)} p\left(z_{j}^{\prime}\right) \wedge f_{\sigma(2)} p\left(z_{j}^{\prime \prime}\right) \wedge f_{\sigma(3)} p\left(z_{j}^{\prime \prime \prime}\right)\right\rangle \\
& =(\ddagger) ;
\end{aligned}
$$

$$
\begin{aligned}
& \frac{1}{\left|\operatorname{Aut}\left(T_{1}\right)\right|}\left\langle v ; \ell_{T_{1}}\left(f_{1}, f_{2}, f_{3}\right)(h)\right\rangle \\
& = \pm \frac{1}{2} \sum_{\sigma \in S_{3}}\left\{\left\langle v ;\left[\left[f_{\sigma(1)} p x^{\prime}, f_{\sigma(2)} p x^{\prime \prime}\right], f_{\sigma(3)} h^{\prime}\right]\right\rangle \pm\left\langle v ;\left[\left[f_{\sigma(1)} p x^{\prime \prime}, f_{\sigma(2)} p x^{\prime}\right], f_{\sigma(3)} h^{\prime}\right]\right\rangle\right\} \\
& \left.= \pm \frac{1}{2} \sum_{\sigma \in S_{3}}\left\{\left\langle u w ;\left[f_{\sigma(1)} p x^{\prime}, f_{\sigma(2)} p x^{\prime \prime}\right] \wedge f_{\sigma(3)} h^{\prime}\right\rangle \pm\left\langle u w ;\left[f_{\sigma(1)} p x^{\prime \prime}, f_{\sigma(2)} p x\right)\right] \wedge f_{\sigma(3)} h^{\prime}\right\rangle\right\} \\
& = \pm \frac{1}{2} \sum_{\sigma \in S_{3}}\left\{\left\langle u ;\left[f_{\sigma(1)} p x^{\prime}, f_{\sigma(2)} p x^{\prime \prime}\right]\right\rangle\left\langle w ; f_{\sigma(3)} p h^{\prime}\right\rangle \pm\left\langle w ;\left[f_{\sigma(1)} p x^{\prime}, f_{\sigma(2)} p x^{\prime \prime}\right]\right\rangle\left\langle u ; f_{\sigma(3)} p h^{\prime}\right\rangle\right. \\
& \left.\quad \pm\left\langle u ;\left[f_{\sigma(1)} p x^{\prime \prime}, f_{\sigma(2)} p x^{\prime}\right]\right\rangle\left\langle w ; f_{\sigma(3)} p h^{\prime}\right\rangle \pm\left\langle w ;\left[f_{\sigma(1)} p x^{\prime \prime}, f_{\sigma(2)} p x^{\prime}\right]\right\rangle\left\langle u ; f_{\sigma(3)} p h^{\prime}\right\rangle\right\} \\
& = \pm \frac{1}{2} \sum_{\sigma \in S_{3}}\left\{\left\langle u^{\prime} u^{\prime \prime} ; f_{\sigma(1)} p x^{\prime} \wedge f_{\sigma(2)} p x^{\prime \prime}\right\rangle\left\langle w ; f_{\sigma(3)} p h^{\prime}\right\rangle\right. \\
& \quad \pm\left\langle w^{\prime} w^{\prime \prime} ; f_{\sigma(1)} p x^{\prime} \wedge f_{\sigma(2)} p x^{\prime \prime}\right\rangle\left\langle u ; f_{\sigma(3)} p h^{\prime}\right\rangle \\
& \quad \pm\left\langle u^{\prime} u^{\prime \prime} ; f_{\sigma(1)} p x^{\prime \prime}, f_{\sigma(2)} p x^{\prime}\right\rangle\left\langle w ; f_{\sigma(3)} p h^{\prime}\right\rangle \\
& \left.\quad \pm\left\langle w^{\prime} w^{\prime \prime} ; f_{\sigma(1)} p x^{\prime \prime}, f_{\sigma(2)} p x^{\prime}\right\rangle\left\langle u ; f_{\sigma(3)} p h^{\prime}\right\rangle\right\}=(\dagger) .
\end{aligned}
$$

\section{Examples and applications}

We will work with models of the components of the mapping space, and for a based map $f: X \rightarrow Y$, we will denote by $\operatorname{map}_{f}^{*}(X, Y)$ the component containing $f$. These $L_{\infty}$-models can be obtained via the process of perturbation and truncation described 
in Sect. 2.1. More explicitly, let $\varphi: \mathcal{L}(\bar{C}) \rightarrow L$ be an $L_{\infty}$-model of $f: X \rightarrow Y$. Then, the composite

$$
\psi: \mathcal{L}(\bar{H}) \longrightarrow \mathcal{L}(\bar{C}) \longrightarrow L
$$

is also a model for $f$. Hence, as explained in [9], the induced degree -1 linear map $\bar{C} \rightarrow L$, which we will keep denoting by $\varphi$, is a Maurer-Cartan element of the $L_{\infty^{-}}$ algebra $\operatorname{Hom}(\bar{C}, L)$, and the induced map $\bar{H} \rightarrow \bar{C} \rightarrow L$ is a Maurer-Cartan element in $\operatorname{Hom}(\bar{H}, L)$. Therefore, the perturbed and truncated $L_{\infty}$-algebras $\operatorname{Hom}(\bar{C}, L)^{(\varphi)}$ and $\operatorname{Hom}(\bar{H}, L)^{(\psi)}$ are $L_{\infty}$-models for $\operatorname{map}_{f}^{*}(X, Y)$.

\subsection{Formality, coformality and mapping spaces}

We recall that $X$ is formal if $H^{*}(X ; \mathbb{Q})$ equipped with the cup product is a differential graded algebra model for the space $X$ or, equivalently, if $X$ has a Quillen minimal model $(\mathbb{L}(V), \partial)$ whose differential is quadratic $\partial=\partial_{2}$. In the same way $Y$ is coformal if $\pi_{*}(\Omega Y) \otimes \mathbb{Q}$ equipped with the Samelson product is a differential graded Lie algebra model for $Y$ or, equivalently, if $Y$ has a Sullivan minimal model $(\Lambda V, d)$ whose differential is quadratic $d=d_{2}$.

The graded vector space which carries the $L_{\infty}$-structure modeling the mapping space is of the form

$$
\operatorname{Hom}\left(H_{*}(X ; \mathbb{Q}), \pi_{*}(\Omega Y) \otimes \mathbb{Q}\right),
$$

although the source and target spaces $X$ and $Y$ are not necessarily formal and coformal. We will now show examples in which the source and/or the target are not formal and coformal.

Example 4.1 Consider the rational space $X$ with Sullivan model $A=(\Lambda(a, b, c), d)$, where $|a|=|b|=3,|c|=5, d a=d b=0$ and $d c=a b$. Note that this space is coformal but not formal as we will see below. Let $Y$ be the rational space with Sullivan model $(\Lambda(x, y, z, t), d)$ where $|x|=4,|y|=7,|z|=10,|t|=16$ and $d x=d y=0$, $d z=x y, d t=y z$.

We will describe the rational homotopy type of map* $(X, Y)$ by studying the $L_{\infty^{-}}$ structure on $\operatorname{Hom}\left(H_{+}(X ; \mathbb{Q}), \pi_{*}(\Omega Y) \otimes \mathbb{Q}\right)$. Note that this mapping space has only one component, the one which contains the constant map since the only morphism of differential graded algebras

$$
\varphi:(\Lambda(x, y, z, t), d) \rightarrow(\Lambda(a, b, c), d)
$$

is the zero morphism $\varphi=0$ by degree reasons.

We have that, as graded Lie algebras,

$$
\pi_{*}\left(\Omega \operatorname{map}^{*}(X, Y)\right) \otimes \mathbb{Q} \cong \operatorname{Hom}\left(H_{+}(X ; \mathbb{Q}), \pi_{*}(\Omega Y) \otimes \mathbb{Q}\right),
$$


since the fact that $\varphi=0$ implies that the bracket $\ell_{1}$ in the $L_{\infty}$-structure given by Theorem 3.2 is zero, or equivalently, the differential in the reduced Brown-Szczarba model has no linear term. However, as we will show the mapping space is not coformal since the differential in its Sullivan model is not purely quadratic.

In particular, we will see that map* $(X, Y)$ splits rationally as

$$
Z \times\left(S^{7} \times S^{7} \times S^{13} \times S^{13}\right)
$$

where $Z$ is the rational space with minimal model

$$
\left(\Lambda\left(a_{1}, b_{1}, a_{2}, b_{2}, a_{4}, b_{4}, z_{5}, x_{8}, y_{8}\right), d\right)
$$

where $d a_{1}=\cdots=d b_{4}=0, d z_{5}=a_{4} b_{2}-b_{4} a_{2}, d x_{8}=-a_{4} a_{1} b_{4}+a_{4}^{2} b_{1}$, and $d y_{8}=-b_{4}^{2} a_{1}+b_{4} b_{1} a_{4}$.

This calculation can be made directly from the Haefliger [21] or the Brown-Szczarba model [4] but, in order to illustrate the homotopy transfer techniques described in this paper, we will make use of Theorem 3.2 instead.

First we describe the $A_{\infty}$-coalgebra structure induced by the higher Massey coproducts on $H_{+}(X ; \mathbb{Q})$. As a graded vector space, $\bar{A}$ is given by

$$
\bar{A}=A^{3} \oplus A^{5} \oplus A^{6} \oplus A^{8} \oplus A^{11}=\langle a, b\rangle \oplus\langle c\rangle \oplus\langle a b\rangle \oplus\langle a c, b c\rangle \oplus\langle a b c\rangle .
$$

The dual coalgebra $\bar{C}=(\bar{A})^{\sharp}$ is

$$
\bar{C}=C_{3} \oplus C_{5} \oplus C_{6} \oplus C_{8} \oplus C_{11}=\langle g, h\rangle \oplus\langle r\rangle \oplus\langle s\rangle \oplus\langle u, v\rangle \oplus\langle w\rangle
$$

with $\delta s=r$ and reduced comultiplications

$$
\begin{aligned}
\bar{\Delta} g=\bar{\Delta} h=\bar{\Delta} r & =0, \\
\bar{\Delta} s & =g \otimes h-h \otimes g, \\
\bar{\Delta} u & =g \otimes r-r \otimes g, \\
\bar{\Delta} v & =h \otimes r-r \otimes h, \\
\bar{\Delta} w & =g \otimes v-v \otimes g-h \otimes u+u \otimes h+s \otimes r+r \otimes s .
\end{aligned}
$$

The decomposition $\bar{C}=S \oplus \delta S \oplus \bar{H}$, where $S=\langle s\rangle, \delta S=\langle r\rangle$ and $\bar{H}=\langle g, h\rangle \oplus$ $\langle u, v\rangle \oplus\langle w\rangle$ induces the homotopy retract

$$
k \bigcirc_{\mathcal{C}}(\bar{C}, \delta) \underset{i}{\stackrel{p}{\gtrless}}(\bar{H}, 0),
$$

where $k(r)=s$. Note that $X$ is not formal since the transferred structure in $\bar{H}$ (which gives rise to the differential on the Quillen minimal model as shown in Theorem 3.1) has non-zero higher order coproducts. Indeed, 


$$
\begin{aligned}
\bar{\Delta}_{3}^{\prime}(u)= & (p \otimes p \otimes \mathrm{id}) \circ(\bar{\Delta} \otimes \mathrm{id}) \circ(k \otimes p) \circ \bar{\Delta} \circ i(u) \\
& +(\mathrm{id} \otimes p \otimes p) \circ(\mathrm{id} \otimes \bar{\Delta}) \circ(p \otimes k) \circ \bar{\Delta} \circ i(u) \\
= & -(p \otimes p \otimes \mathrm{id})(\bar{\Delta}(s) \otimes g)+(\mathrm{id} \otimes p \otimes p)(g \otimes \bar{\Delta}(s)) \\
= & (g \otimes g \otimes h)-2(g \otimes h \otimes g)+(h \otimes g \otimes g) .
\end{aligned}
$$

On the other hand, the model for $Y$ is of the form $\mathcal{C}^{*}(L)=(\Lambda(x, y, z, t), d)$ for some $L_{\infty}$-algebra $L$ with $\langle x, y, z, t\rangle \cong(s L)^{\sharp}$, and brackets induced by the differential $d$ by formula (2.1). We write $L=\left\langle x^{\prime}, y^{\prime}, z^{\prime}, t^{\prime}\right\rangle$ with $\left|x^{\prime}\right|=3,\left|y^{\prime}\right|=6,\left|z^{\prime}\right|=9$, $\left|t^{\prime}\right|=15$.

With the above data we can define a homotopy retract

$$
k^{*} \bigcirc \operatorname{Hom}\left(\bar{C},\left\langle x^{\prime}, y^{\prime}, z^{\prime}, t^{\prime}\right\rangle\right) \underset{p^{*}}{\stackrel{i^{*}}{\rightleftarrows}} \operatorname{Hom}\left(\bar{H},\left\langle x^{\prime}, y^{\prime}, z^{\prime}, t^{\prime}\right\rangle\right)
$$

where $\operatorname{Hom}\left(\bar{H},\left\langle x^{\prime}, y^{\prime}, z^{\prime}, t^{\prime}\right\rangle\right) \cong \operatorname{Hom}\left(H_{+}(X ; \mathbb{Q}), \pi_{*}(\Omega Y) \otimes \mathbb{Q}\right)$ as graded vector spaces. Then we have that $\operatorname{Hom}\left(H_{+}(X ; \mathbb{Q}), \pi_{*}(\Omega Y) \otimes \mathbb{Q}\right)=K_{*}$, with

$$
K_{*}=K_{-8} \oplus K_{-5} \oplus K_{-2} \oplus K_{0} \oplus K_{1} \oplus K_{3} \oplus K_{4} \oplus K_{6} \oplus K_{7} \oplus K_{12},
$$

where $K_{-8}=\left\langle f_{w}^{x^{\prime}}\right\rangle$ with $f_{w}^{x^{\prime}}(w)=x^{\prime}$ and $f_{w}^{x^{\prime}}(\Phi)=0$ if $\Phi$ is any other basis element, and

$$
\begin{aligned}
& K_{-5}=\left\langle f_{w}^{y^{\prime}}, f_{u}^{x^{\prime}}, f_{v}^{x^{\prime}}\right\rangle, K_{-2}=\left\langle f_{u}^{y^{\prime}}, f_{v}^{y^{\prime}}, f_{w}^{z}\right\rangle, K_{0}=\left\langle f_{g}^{x^{\prime}}, f_{h}^{x^{\prime}}\right\rangle, K_{1}=\left\langle f_{u}^{z^{\prime}}, f_{v}^{z^{\prime}}\right\rangle, \\
& K_{3}=\left\langle f_{g}^{y^{\prime}}, f_{h}^{y^{\prime}}\right\rangle, K_{4}=\left\langle f_{w}^{t^{\prime}}\right\rangle, K_{6}=\left\langle f_{g}^{z^{\prime}}, f_{h}^{z^{\prime}}\right\rangle, K_{7}=\left\langle f_{u}^{t^{\prime}}, f_{v}^{t^{\prime}}\right\rangle, K_{12}=\left\langle f_{g}^{t^{\prime}}, f_{h}^{t^{\prime}}\right\rangle .
\end{aligned}
$$

The higher brackets can be computed using the explicit formula given in Theorem 2.3(ii), but the only Maurer-Cartan element will be the zero element since there is only one component. So, in order to compute the model of the mapping space we must only discard the elements of negative degree. We give some explicit computations. In order to compute $\ell_{2}^{\prime}$ we have to consider the tree

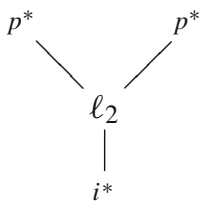

For the element $w$ we have that

$$
\begin{aligned}
\ell_{2}^{\prime}\left(f_{g}^{y^{\prime}}, f_{v}^{z^{\prime}}\right)(w)= & \left(\ell_{2}\right)_{L} \circ\left(\left(f_{g}^{y^{\prime}} \circ p\right) \otimes\left(f_{v}^{z^{\prime}} \circ p\right)\right) \circ \bar{\Delta}(w) \\
= & \left(\ell_{2}\right)_{L} \circ\left(\left(f_{g}^{y^{\prime}} \circ p\right) \otimes\left(f_{v}^{z^{\prime}} \circ p\right)\right)(g \otimes v-v \otimes g-h \otimes u \\
& +u \otimes h+s \otimes r+r \otimes s) \\
= & \left(\ell_{2}\right)_{L}\left(y^{\prime}, z^{\prime}\right)=t^{\prime},
\end{aligned}
$$


and it is easy to check that $\ell_{2}^{\prime}\left(f_{g}^{y^{\prime}}, f_{v}^{z^{\prime}}\right)$ vanishes in the rest of basis elements. Hence $\ell_{2}^{\prime}\left(f_{g}^{y^{\prime}}, f_{v}^{z^{\prime}}\right)=f_{w}^{t^{\prime}}$. For computing $\ell_{3}^{\prime}$ we have to use the tree

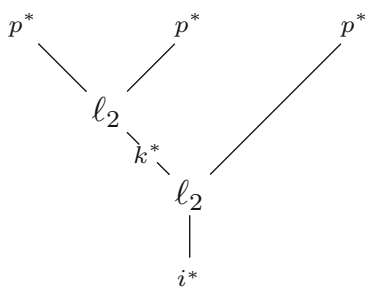

For the element $u$, we have that

$$
\begin{aligned}
\ell_{3}^{\prime}\left(f_{g}^{y^{\prime}}, f_{h}^{x^{\prime}}, f_{h}^{y^{\prime}}\right)(u) & =i^{*} \ell_{2}\left(k^{*}\left(\ell_{2}\left(p^{*} f_{g}^{y^{\prime}}, p^{*} f_{h}^{x^{\prime}}\right)\right), p^{*} f_{h}^{y^{\prime}}\right)(u) \\
& =\left(\ell_{2}\right)_{L} \circ\left(k^{*}\left(\ell_{2}\left(p^{*} f_{g}^{y^{\prime}}, p^{*} f_{h}^{x^{\prime}}\right)\right) \otimes p^{*} f_{h}^{y^{\prime}}\right) \circ \bar{\Delta}(u) \\
& =\left(\ell_{2}\right)_{L} \circ\left(k^{*}\left(\ell_{2}\left(p^{*} f_{g}^{y^{\prime}}, p^{*} f_{h}^{x^{\prime}}\right)\right) \otimes p^{*} f_{h}^{y^{\prime}}\right)(g \otimes r-r \otimes g) \\
& =\left(\ell_{2}\right)_{L}\left(\ell_{2}\left(p^{*} f_{g}^{y^{\prime}}, p^{*} f_{h}^{x^{\prime}}\right) k(r), f_{g}^{y^{\prime}}(g)\right) \\
& =\left(\ell_{2}\right)_{L}\left(\ell_{2}\left(p^{*} f_{g}^{y^{\prime}}, p^{*} f_{h}^{x^{\prime}}\right)(s), f_{g}^{y^{\prime}}(g)\right) \\
& =-\left(\ell_{2}\right)_{L}\left(\left(\ell_{2}\right)_{L}\left(f_{g}^{y^{\prime}}(g), f_{h}^{x^{\prime}}(h), y^{\prime}\right)\right. \\
& =-\left(\ell_{2}\right)_{L}\left(\left(\ell_{2}\right)_{L}\left(y^{\prime}, x^{\prime}\right), y^{\prime}\right)=\left(\ell_{2}\right)_{L}\left(z^{\prime}, y^{\prime}\right)=-t^{\prime} .
\end{aligned}
$$

Again, it is easy to check that $\ell_{3}^{\prime}\left(f_{g}^{y^{\prime}}, f_{h}^{x^{\prime}}, f_{h}^{y^{\prime}}\right)$ vanishes in the rest of basis elements. Hence $\ell_{3}^{\prime}\left(f_{g}^{y^{\prime}}, f_{h}^{x^{\prime}}, f_{h}^{y^{\prime}}\right)=-f_{u}^{t^{\prime}}$.

We can compute the cochain functor of this $L_{\infty}$-algebra

$$
\mathcal{C}^{*}(\operatorname{Hom}(\bar{H}, L))=\left(\Lambda\left((s L)^{\sharp} \otimes \bar{H}\right), d\right),
$$

and the rational homotopy type of map* $(X, Y)$ is modeled by

$$
\left(\Lambda\left((s L)^{\sharp} \otimes \bar{H}\right)^{>0}, \widehat{d}\right)
$$

as described in Sect. 2.3, which coincides with the reduced Brown-Szczarba model. Explicitly, $\left((s L)^{\sharp} \otimes \bar{H}\right)^{>0}$ is concentrated in degrees $1,2,4,5,7,8$ and 13 ,

$$
\begin{aligned}
\left((s L)^{\sharp} \otimes \bar{H}\right)^{1} & =\langle x \otimes g, x \otimes h\rangle,\left((s L)^{\sharp} \otimes \bar{H}\right)^{2}=\langle z \otimes u, z \otimes v\rangle, \\
\left((s L)^{\sharp} \otimes \bar{H}\right)^{4} & =\langle y \otimes g, y \otimes h\rangle, \quad\left((s L)^{\sharp} \otimes \bar{H}\right)^{5}=\langle t \otimes w\rangle, \\
\left((s L)^{\sharp} \otimes \bar{H}\right)^{7} & =\langle z \otimes g, z \otimes h\rangle, \quad\left((s L)^{\sharp} \otimes \bar{H}\right)^{8}=\langle t \otimes u, t \otimes v\rangle, \\
\left((s L)^{\sharp} \otimes \bar{H}\right)^{13} & =\langle t \otimes g, t \otimes h\rangle,
\end{aligned}
$$


and the differentials are given by

$$
\begin{aligned}
\widehat{d}(x \otimes g) & =\widehat{d}(x \otimes h)=\widehat{d}(z \otimes u)=\widehat{d}(z \otimes v)=\widehat{d}(y \otimes g)=\widehat{d}(y \otimes h) \\
& =\widehat{d}(z \otimes g)=\widehat{d}(z \otimes h)=\widehat{d}(t \otimes g)=\widehat{d}(t \otimes h)=0, \\
\widehat{d}(t \otimes w) & =(y \otimes g)(z \otimes v)-(y \otimes h)(z \otimes u), \\
\widehat{d}(t \otimes u) & =-(y \otimes g)(x \otimes g)(y \otimes h)+(y \otimes g)^{2}(x \otimes h), \\
\widehat{d}(t \otimes v) & =-(y \otimes h)^{2}(x \otimes g)+(y \otimes h)(x \otimes h)(y \otimes g) .
\end{aligned}
$$

Thus, we obtain the rational equivalence

$$
\operatorname{map}^{*}(X, Y) \simeq \mathbb{Q} Z \times\left(S^{7} \times S^{7} \times S^{13} \times S^{13}\right) .
$$

\section{2 $H$-space structures}

Another interesting question is to determine whether a mapping space is of the rational homotopy type of an $H$-space, that is, it has a Sullivan minimal model with zero differential, in terms of the source and target spaces.

Recall that for a space $X$, the differential length $\mathrm{dl}(X)$ is the least integer $n$ such that there is a non-trivial Whitehead product of order $n$ on $\pi_{*}(X) \otimes \mathbb{Q}$. This number coincides with the least $n$ for which the $n$-th part of the differential of the Sullivan minimal model of $X$ is non trivial; see [1]. If there is not such an $n$, then $\operatorname{dl}(X)=\infty$. Dually, the bracket length $\mathrm{bl}(X)$ is the length of the shortest non-zero iterated bracket in the differential of the Quillen minimal model of $X$. If the differential is zero, then $\mathrm{bl}(X)=\infty$.

The rational cone length $\operatorname{cl}(X)$ is the least integer $n$ such that $X$ has the rational homotopy type of an $n$-cone; see [15, p. 359]. The rational Whitehead length $\mathrm{Wl}(X)$ is the length of the longest non-zero iterated Whitehead bracket in $\pi_{\geq 2}(X) \otimes \mathbb{Q}$. In particular, if $\mathrm{Wl}(X)=1$, then all Whitehead products vanish.

In [16, Theorem 2] a necessary condition, in terms of the Toomer invariant, is given in order to ensure that the component of the constant map is an $H$-space. In [7, Theorem 4] it was proved that if $\operatorname{cl}(X)<\mathrm{dl}(Y)$ then all the components $\operatorname{map}_{f}^{*}(X, Y)$ are rationally $H$-spaces. A dual result (in the sense of Eckmann-Hilton) was given in [5, Theorem 1.4(2)], by assuming that $Y$ is a coformal space. In this case, if $\mathrm{Wl}(Y)<$ $\mathrm{bl}(X)$ then all the components $\operatorname{map}_{f}^{*}(X, Y)$ are again rationally $H$-spaces. In this paper we formulate a variant of this last result that does not implicitly assume the coformality of $Y$.

Theorem 4.2 If $\mathrm{cl}(X)=2$ and $\mathrm{Wl}(Y)<\mathrm{bl}(X)$, then all the components of the mapping space map* $(X, Y)$ are rationally $H$-spaces.

Proof Let $L$ be the minimal $L_{\infty}$-model of $Y$. Following [14] or [15, Theorem 29.1], and since $\operatorname{cl}(X)=2$, we may choose $C$ a coalgebra model of $X$ with conilpotence 2, that is, such that the iterated coproducts of length greater or equal than 2 are zero. Consider the $L_{\infty}$-model $\operatorname{Hom}(H, L)^{(\phi)}$ of the component corresponding to $\phi$ given in 
Theorem 3.2. In order to prove that $\operatorname{map}_{f}^{*}(X, Y)$ is an $H$-space we will show that all brackets $\ell_{k}^{\phi}$ for $k \geq 2$ vanish, and thus $\mathcal{C}^{*}\left(\operatorname{Hom}(H, L)^{(\phi)}\right)$ is of the form $\left(\Lambda S, d_{1}\right)$, for which $\left(\Lambda H\left(S, d_{1}\right), 0\right)$ is a Sullivan minimal model. It is enough to check that $\ell_{k}^{\prime}=0$ for all $k$ since this implies that $\ell_{k}^{\prime \phi}=0$ for all $k$.

Now, all the brackets are represented by summations on trees. But due to the conilpotence condition on $C$ only the binary ones contribute to the brackets. Then as we have pointed in Remark 3.1, the operations given by these trees are defined in terms of the differential in the minimal model of $X$. Since the bracket length is the length of the shortest non-zero iterated bracket appearing in the differential of the Quillen minimal model, the corresponding binary tree is expressed by an iterated bracket of this length in $L$, and hence it must vanish.

We illustrate the last part of the proof by showing that $\ell_{4}^{\prime}=0$. This operation is defined as a sum of maps whose terms are indexed by the following five trees:
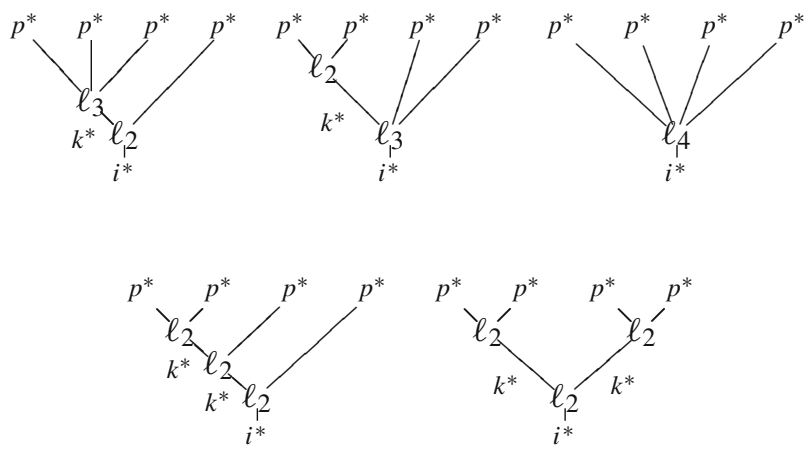

Note that the first three trees do not contribute to $\ell_{4}^{\prime}$ since they are not binary. For example, for the second tree we have that

$$
\begin{aligned}
& i^{*} \ell_{3}\left(k^{*} \ell_{2}\left(p^{*} f_{1}, p^{*} f_{2}\right), p^{*} f_{3}, p^{*} f_{4}\right)(h) \\
& \quad=[-,-,-]_{L} \circ\left(k^{*} \ell_{2}\left(p^{*} f_{1}, p^{*} f_{2}\right) \otimes p^{*} f_{3} \otimes p^{*} f_{4}\right) \circ \bar{\Delta}^{(2)}(h)
\end{aligned}
$$

is zero. Indeed, $\bar{\Delta}^{(2)}=(\Delta \otimes \mathrm{id}) \circ \Delta: C \rightarrow C \otimes C \otimes C$ is zero since $\operatorname{cl}(X)=2$.

The last two trees do not contribute to $\ell_{4}^{\prime}$ either, since $\mathrm{Wl}(Y)<\mathrm{bl}(X)$. Thus, for example, for any $h \in \bar{H}$, the fourth tree yields

$$
i^{*} \ell_{2}\left(k^{*} \ell_{2}\left(k^{*} \ell_{2}\left(p^{*} f_{1}, p^{*} f_{2}\right), p^{*} f_{3}\right), p^{*} f_{4}\right)(h) .
$$

A generic term in this expression is given by

$$
\left.\sum_{i}\left[\left[f_{1} p x_{i}^{\prime}, f_{2} p x_{i}^{\prime \prime}\right]_{L}, f_{3} p y_{j}^{\prime \prime}\right]_{L}, f_{4} p z_{l}^{\prime \prime}\right]_{L}
$$

where $\bar{\Delta}(h)=\sum_{l} z_{l}^{\prime} \otimes z_{l}^{\prime \prime}, z_{l}^{\prime}=\delta a$ and hence $k z_{l}^{\prime}=a, \bar{\Delta}(a)=\sum_{j} y_{j}^{\prime} \otimes y_{j}^{\prime \prime}, y_{j}^{\prime}=\delta a^{\prime}$ and hence $k y_{j}^{\prime}=a^{\prime}$, and $\bar{\Delta}\left(a^{\prime}\right)=\sum_{i} x_{i}^{\prime} \otimes x_{i}^{\prime \prime}$. If $p x_{i}^{\prime}, p x_{i}^{\prime \prime}, p y_{j}^{\prime \prime}, p z_{l}^{\prime \prime} \neq 0$, then by 
the recursive formula (3.2) of Proposition 3.1, the term

$$
\left.\left[\left[s^{-1} p x_{i}^{\prime}, s^{-1} p x_{i}^{\prime \prime}\right], s^{-1} p y_{j}^{\prime}\right], s^{-1} p z_{l}^{\prime \prime}\right]
$$

appears in the expression of $\partial s^{-1} h$, where $\partial$ is the differential of the Quillen minimal model of $X$. Therefore, (4.1) must be zero, since $\mathrm{Wl}(Y)<\operatorname{bl}(X)$.

Remark 4.3 We can give an alternative proof of Theorem 4.2 by relying on [5, Theorem 1.4(2)], where the same result is obtained, but under the assumptions $\mathrm{Wl}(Y)<\mathrm{bl}(X)$ and coformality of the target space $Y$. Indeed, let $L$ be a minimal $L_{\infty}$-model of $Y$ and denote by $L^{\text {cof }}$ the differential graded Lie algebra obtained from $L$ by discarding all higher brackets except the binary one. The graded Lie algebra $L^{\text {cof }}$ represents a coformal rational space $Y^{\text {cof }}$ such that $\mathrm{Wl}(Y)=\mathrm{Wl}\left(Y^{\text {cof }}\right)$. Let $C$ be a coalgebra model of $X$ such that $\bar{C}$ has conilpotence $\operatorname{cl}(X)=2$. Then the convolution $L_{\infty}$-algebra $\operatorname{Hom}(\bar{C}, L)$ is isomorphic to the convolution differential graded Lie algebra $\operatorname{Hom}\left(\bar{C}, L^{\text {cof }}\right)$. Therefore $\operatorname{map}^{*}(X, Y) \simeq \operatorname{map}^{*}\left(X, Y^{\text {cof }}\right)$.

Example 4.4 Let $X=S_{a}^{7} \vee S_{b}^{7} \cup_{\gamma} e^{20}$, let $\alpha, \beta \in \pi_{7}(X)$ be the elements represented by $S_{a}^{7}$ and $S_{b}^{7}$, respectively, and let $\gamma=[\alpha,[\alpha, \beta]] \in \pi_{19}(X)$. Then, the Quillen minimal model for $X$ is $(\mathbb{L}(W), \partial)$, where $W=\langle a, b, c\rangle,|a|=|b|=6$ and the only non-zero differential is $\partial c=[a,[a, b]]$. Hence, it is clear that $\operatorname{bl}(X)=3$. Moreover, $\operatorname{cl}(X)=2$ since $W=W_{0} \oplus W_{1}$ with $\partial W_{0}=0$ and $\partial W_{1} \subseteq \mathbb{L}\left(W_{0}\right)$; see, for example, [15, Theorem 29.1].

Let $Y$ be the space with Sullivan minimal model of the form $(\Lambda(u, v, w), d),|u|=$ $2,|v|=4,|w|=7, d u=d v=0, d w=u^{4}+v^{2}$.

Therefore, $Y$ is a non-coformal space with $\mathrm{dl}(Y)=\mathrm{Wl}(Y)=2$. Moreover, there are non null-homotopic maps from $X$ to $Y$. For example, $\phi: \Lambda V \rightarrow \mathcal{C}^{*}(\mathbb{L}(a, b, c))=$ $\Lambda s \mathbb{L}(a, b, c)^{\sharp}$, defined by $\phi(u)=\phi(v)=0$ and $\phi(w)=s a^{\sharp}$.

Then, by Theorem 4.2 , every component of the mapping space map* $(X, Y)$ is rationally an $H$-space. This example provides a situation where we cannot apply neither [7, Theorem 4], since $\operatorname{cl}(X)=\mathrm{dl}(Y)$, nor [5, Theorem 1.4(2)], since $Y$ is not coformal. Also [16, Theorem 2] does not provide any information for the component of a non-constant map.

\section{References}

1. Andrews, P., Arkowitz, M.: Sullivan's minimal models and higher order Whitehead products. Can. J. Math. 30(5), 961-982.0 (1978)

2. Berciano, A., Real, P.: $A_{\infty}$-coalgebra structure maps that vanish on $H_{*}\left(K(\pi, n), \mathbb{Z}_{p}\right)$. Forum Math. 22(2), 357-378 (2010)

3. Berglund, A.: Rational homotopy theory of mapping spaces via Lie theory for $L_{\infty}$ algebras. arXiv:1110.6145 (preprint)

4. Brown, E.H., Szczarba, R.H.: On the rational homotopy type of function spaces. Trans. Am. Math. Soc. 349, 4931-4951 (1997)

5. Buijs, U.: An explicit $L_{\infty}$ structure for the components of mapping spaces. Topol. Appl. 159(3), 721-732 (2012)

6. Buijs, U.: Upper bounds for the Whitehead length of mapping spaces. Homotopy theory of function spaces and related topics, 43-53. Contemp. Math. 519. Amer. Math. Soc., Providence (2010) 
7. Buijs, U., Murillo, A.: The rational homotopy Lie algebra of function spaces. Comment. Math. Helv. 83, 723-739 (2008)

8. Buijs, U., Murillo, A.: Algebraic models of non-connected spaces and homotopy theory of $L_{\infty}$ algebras. Adv. Math. 236, 60-91 (2013)

9. Buijs, U., Félix, Y., Murillo, A.: $L_{\infty}$ rational homotopy theory of mapping spaces. Rev. Mat. Complut. 26(2), 573-588 (2013)

10. Buijs, U., Félix, Y., Murillo, A.: $L_{\infty}$ models of mapping spaces. J. Math. Soc. Japan 63(2), 503-524 (2011)

11. Buijs, U., Gutiérrez, J.J., Murillo, A.: Derivations, the Lawrence-Sullivan interval and the FiorenzaManetti mapping cone. Math. Z. 273(3-4), 981-997 (2013)

12. Chas, M., Sullivan, D.: String topology. arXiv:math/9911159 (preprint)

13. Cheng, X.Z., Getzler, E.: Transferring homotopy commutative algebraic structures. J. Pure Appl. Algebra 212(11), 2535-2542 (2008)

14. Cornea, O.: Cone length and Lusternik-Schnirelmann category. Topology 33, 95-111 (1994)

15. Félix, Y., Halperin, S., Thomas, J.C.: Rational homotopy theory. Graduate Texts in Mathematics, vol. 205. Spinger, Berlin (2000)

16. Félix, Y., Tanré, D.: $H$-space structure on pointed mapping spaces. Algebr. Geom. Topol. 5, 713-724 (2005)

17. Fukaya, K.: Deformation theory, homological algebra and mirror symmetry. Geometry and physics of branes (Como 2001), pp. 121-209. Ser. High Energy Phys. Cosmol. Gravit, IOP, Bristol (2003)

18. Getzler, E.: Lie theory for nilpotent $L_{\infty}$ algebras. Ann. Math. 170(1), 271-301 (2009)

19. Gugenheim, V.K.A.M., Stasheff, J.D.: On perturbations and $A_{\infty}$-structures. Bull. Soc. Math. Belg. Sér. A 38, 237-246 (1986)

20. Gugenheim, V.K.A.M., Lambe, L.A., Stasheff, J.D.: Perturbation theory in differential homological algebra II. Illinous J. Math. 35(3), 357-373 (1991)

21. Haefliger, A.: Rational homotopy of the space of sections of a nilpotent bundle. Trans. Am. Math. Soc. 273, 609-620 (1982)

22. Huebschmann, J., Kadeishvili, T.: Small models for chain algebras. Math. Z. 207(2), 245-280 (1991)

23. Kadeishvili, T.: The algebraic structure in the homology of an $A(\infty)$-algebra. Soobshch. Akad. Nauk Gruzin. SSR 108(1982)(2), 249-252 (1983)

24. Kontsevich, M.: Deformation quantization of Poisson manifolds. Lett. Math. Phys. 66(3), 157-216 (2003)

25. Kontsevich, M., Soibelman, Y.: Deformations of algebras over operads and Deligne's conjecture. In: Dito, G., Sternheimer, D. (eds) Conférence Moshé Flato 1999, vol. I (Dijon 1999), pp. 255-307. Kluwer Acad. Publ., Dordrecht (2000)

26. Kontsevich, M., Soibelman, Y.: Homological mirror symmetry and torus fibrations. Symplectic geometry and mirror symmetry (Seoul 2000), pp. 203-263. World Sci. Publ, River Edge (2000)

27. Lazarev, A.: Maurer-Cartan moduli and models for function spaces. Adv. Math. 235, 296-320 (2013)

28. Loday, J.L., Vallette, B.: Algebraic Operads. Grundlehren Math. Wiss., vol. 346. Springer, Berlin (2012)

29. Lupton, G., Smith, S.B.: Whitehead products in function spaces: Quillen model formulae. J. Math. Soc. Japan 62(1), 49-81 (2010)

30. Merkulov, S.A.: Strong homotopy algebras of a Kähler manifold. Int. Math. Res. Not. 1999(3), 153-164 (1999)

31. Neisendorfer, J., Miller, T.: Formal and coformal spaces. Ill. J. Math. 22(4), 565-580 (1978)

32. Schlessinger, M., Stasheff, J.D.: The Lie algebra structure of tangent cohomology and deformation theory. J. Pure Appl. Algebra 38(2-3), 313-322 (1985)

33. Sullivan, D.: Infinitesimal computations in topology. Publ. Math. Inst. Hautes Etudes Sci. 47, 269-331 (1978) 